ARTICLE

https://doi.org/10.1038/s41467-019-13572-w

\title{
TRPML1 links lysosomal calcium to autophagosome biogenesis through the activation of the CaMKK $\beta$ / VPS34 pathway
}

\author{
A. Scotto Rosato ${ }^{1,2,9}$, S. Montefusco ${ }^{1,9}$, C. Soldati ${ }^{1}$, S. Di Paola (i) ${ }^{1}$, A. Capuozzo ${ }^{1}$, J. Monfregola1, E. Polishchuk 1 , \\ A. Amabile ${ }^{3,4}$, C. Grimm², A. Lombardo (1) ${ }^{3,4}$, M.A. De Matteis (1) 1,5, A. Ballabio (1) 1,6,7,8 \& D.L. Medina (1) 1,6*
}

The lysosomal calcium channel TRPML1, whose mutations cause the lysosomal storage disorder (LSD) mucolipidosis type IV (MLIV), contributes to upregulate autophagic genes by inducing the nuclear translocation of the transcription factor EB (TFEB). Here we show that TRPML1 activation also induces autophagic vesicle ( $A \mathrm{~V}$ ) biogenesis through the generation of phosphatidylinositol 3-phosphate (PI3P) and the recruitment of essential PI3P-binding proteins to the nascent phagophore in a TFEB-independent manner. Thus, TRPML1 activation of phagophore formation requires the calcium-dependent kinase CaMKK $\beta$ and AMPK, which increase the activation of ULK1 and VPS34 autophagic protein complexes. Consistently, cells from MLIV patients show a reduced recruitment of PI3P-binding proteins to the phagophore during autophagy induction, suggesting that altered AV biogenesis is part of the pathological features of this disease. Together, we show that TRPML1 is a multistep regulator of autophagy that may be targeted for therapeutic purposes to treat LSDs and other autophagic disorders.

\footnotetext{
${ }^{1}$ Telethon Institute of Genetics and Medicine (TIGEM), Pozzuoli, Naples, Italy. ${ }^{2}$ Faculty of Medicine, Walther Straub Institute of Pharmacology and Toxicology, Ludwig-Maximilians-Universität, Munich, Germany. ${ }^{3}$ Telethon Institute for Gene Therapy (SR-Tiget), Division of Regenerative Medicine, Stem Cells, and Gene Therapy, IRCCS San Raffaele Scientific Institute, Milan, Italy. ${ }^{4}$ Vita-Salute San Raffaele University, 20132 Milan, Italy. ${ }^{5}$ Department of Molecular Medicine and Medical Biotechnology, Federico II University, Naples, Italy. ${ }^{6}$ Medical Genetics Unit, Department of Medical and Translational Science, Federico II University, Naples, Italy. ${ }^{7}$ Baylor College of Medicine, Houston, Texas, USA. ${ }^{8}$ Jan and Dan Duncan Neurological Research Institute, Texas Children's Hospital, Houston, Texas, USA. ${ }^{9}$ These authors contributed equally: A. Scotto Rosato, S. Montefusco *email: medina@tigem.it
} 
C alcium is a universal second messenger that plays fundamental roles in cellular physiology. To allow rapid mobilization upon specific stimuli, calcium is kept at extremely low levels in the cytoplasm by its compartmentalization in organelles during resting conditions ${ }^{1}$. Endoplasmic reticulum (ER) and mitochondria ${ }^{2}$ are recognized as major cellular calcium stores; however, recent evidence indicates that lysosomal calcium also plays an important role in a variety of cellular processes $^{3}$. Furthermore, impairment of lysosomal calcium homeostasis has been implicated in several human diseases such as lysosomal storage disorders (LSDs) ${ }^{4-6}$, neurodegenerative diseases $^{7-11}$, muscular dystrophy, and cancer ${ }^{12-14}$. The nonselective cation channel TRPML1 is a major calcium-release channel on the lysosomal membrane ${ }^{3}$. TRPML1 activity is involved in a variety of membrane-trafficking processes such as lysosome to trans-Golgi-network retrograde trafficking, autophagic vesicle (AV)-lysosome fusion, lysosome reformation, and lysosomal exocytosis ${ }^{3}$. Mutations in TRPML1 cause mucolipidosis type IV (MLIV: OMIM 252650), an autosomal recessive LSD characterized by psychomotor alterations, corneal opacities, and achlorhydria ${ }^{15-17}$. Cells from MLIV patients present defects in macroautophagy that are characterized by the accumulation of autophagic markers such as LC3 and p62 $2^{18-20}$. Although the autophagic defects in MLIV, as well as in other LSDs, have been interpreted as the consequence of a global lysosomal dysfunction ${ }^{21}$, more specific mechanisms have not been identified.

Recent studies suggest that TRPML1 also plays a major role in lysosomal signaling during nutrient deprivation. Lysosomal calcium release through TRPML1 promotes the dephosphorylation of TFEB by the phosphatase calcineurin, thus inducing TFEB nuclear translocation and the consequent transcriptional activation of lysosomal and autophagic genes ${ }^{22,23}$. Thus, in addition to mediating the fusion of autophagosomes with lysosomes ${ }^{19,24,25}$, TRPML1 regulates autophagy by controlling the activity of the master transcriptional regulator of autophagy TFEB. Interestingly, TRPML1 and TFEB are involved in a feedback loop by which TRPML1 is at the same time a controller of TFEB activity and a downstream transcriptional target of TFEB and major effector of TFEB biological activity ${ }^{23,26}$. Here, by using genetic and pharmacological approaches to modulate TRPML1 activity, we show that TRPML1 can regulate autophagy by an additional mechanism, which is not transcriptional and is independent of TFEB. Thus, TRPML1 can rapidly induce AV biogenesis through a signaling pathway that involves the activation of calcium/calmodulin-dependent protein kinase kinase $\beta$ (CaMKK $\beta$ ) and AMP-activated protein kinase (AMPK), the induction of the Beclin1/VPS34 autophagic complex, and the generation of phosphatidylinositol 3-phosphate (PI3P). This mechanism is pathophysiologically relevant, as MLIV patient cells show a reduced recruitment of PI3P-binding proteins to the phagophore during autophagy induction. Thus, our data identify TRPML1 as a multistep regulator of autophagy and a global controller of cell metabolism.

\section{Results}

TRPML1 induces AV formation independently of TFEB. We have recently shown that TRPML1 activity induces TFEB nuclear translocation through the activation of the phosphatase calcineurin and consequent dephosphorylation of TFEB during starvation $^{23}$. This ability of TRPML1 to activate TFEB results in an enhanced expression of lysosomal and autophagic genes, and induction of autophagy. Consistently, silencing of TFEB reduces the effect of TRPML1 on autophagy induction ${ }^{23}$. However, the production of a functional protein from gene transcription to its translation can take significantly more time than calcium mobilization $^{1,27}$. Thus, we asked whether the acute activation of TRPML1 could also contribute to the regulation of the autophagic pathway in a transcription-independent manner. Therefore, we analyzed critical steps of the autophagic pathway at several time points after pharmacological induction of TRPML1 channel activity using two synthetic agonists, MK6-83 and ML-SA1 5,28,29. We found that both agonists increase LC3 puncta formation at all time points tested, 30 and $90 \mathrm{~min}$ (Fig. 1a). Also, we found that MK6-83-mediated elevation of LC3 puncta formation was further increased in cells overexpressing TRPML1 (Supplemen-

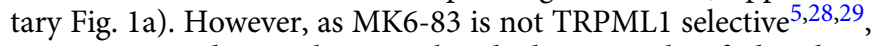
we investigated its selectivity by depleting each of the three channels belonging to the TRPML's family. We found that MK683 activity was fully inhibited in cells depleted of TRPML1, by using both genome editing or acute silencing, but not in cells depleted of TRPML2 or TRPML3, indicating that MK6-83 can induce AV formation through TRPML1 independently of the other channels (Supplementary Fig. 1b-e). In contrast to the more ubiquitous expression of TRPML1, the expression and subcellular localization of the other members of this family is tissue-specific and not restricted to the lysosomal compartment ${ }^{20}$. By using expression vectors carrying either TRPML2 or TRPML3, we found that TRPML3 overexpression, but not TRPML2, was able to increase LC3 puncta formation (Supplementary Fig. 1f). Similar results were obtained by testing the ability of synthetic agonist of either TRPML2 or TRPML3, ML2-SA1, and SN-2 $2^{30,31}$, respectively. In accordance with our TRPML2 overexpression data, ML2-SA1 was not able to induce LC3 puncta formation (Supplementary Fig. 1g). Conversely, SN-2 was able to weakly induce LC3 puncta formation in both wild-type (WT) and TRPML1-depleted cells (Supplementary Figs. $1 \mathrm{~g}$ and 2a, b), indicating that TRPML3 may regulate autophagy independently of other TRPML members, most likely in tissues where it is highly expressed $^{20}$.

Focusing on TRPML1, we also observed a further elevation of endogenous LC3 lipidation in the presence of MK6-83 and the lysosomal inhibitor bafilomycin A1, suggesting that TRPML1 activation induces $\mathrm{AV}$ maturation and autophagy activation (Fig. 1b, c). Similarly, overexpression of TRPML1 increased LC3II lipidation and this effect was enhanced by bafilomycin A1 treatment (Supplementary Fig. 2c). Conversely, cells co-treated with MK6-83 and the TRPML1 inhibitor ML-SI3 ${ }^{32}$ showed a reduction in LC3II formation compared with MK6-83 treatment alone (Supplementary Fig. 2d). Consistently, we found that both pharmacological activation of TRPML1 and TRPML1 overexpression increased the AV-lysosome fusion as measured by the colocalization of LC3 with the lysosomal membrane protein LAMP1 (Fig. 1d and Supplementary Fig. 2e), an effect that was suppressed, at least partially, by the inhibitor of AV-lysosomal fusion vinblastine as well as by the silencing of the autophagic SNARE protein syntaxin-1733,34 (Supplementary Fig. 3a-c). Notably, we also found some LAMP1-positive vesicles stained with LC3 even after AV-lysosomal fusion inhibition, suggesting that TRPML1 activation may also induce lipidation of other structures, most likely endolysosomal vesicles, a phenomenon that has been previously observed in cells treated with other autophagy modulators ${ }^{35}$.

Electron microscopy analysis of a stable U2OS cell line overexpressing LC3-GFP also showed an increase in the number of autolysosomes upon MK6-83 treatment (Supplementary Fig. 4a). Similarly, an increase in the number of autolysosomes (enhanced green fluorescent protein (EGFP)-negative and red fluorescent protein (RFP)-positive) was observed in a stable HeLa cell line overexpressing the autophagic reporter RFP-EGFP-LC3 ${ }^{33}$ (Fig. 1e). Together, these data indicate that activation of TRPML1 
a

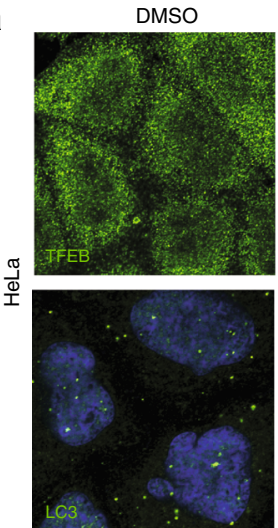

MK6-83 -30 min
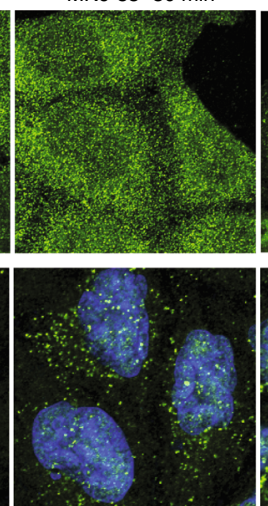

MK6-83 -90 min
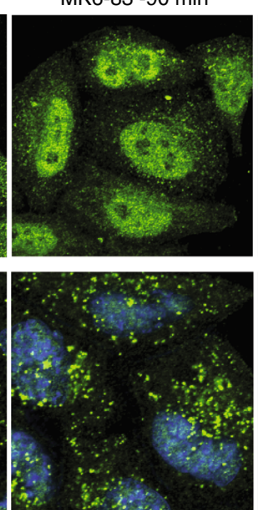

ML-SA1 -30 min
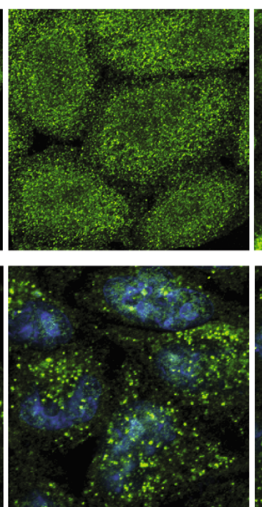

C
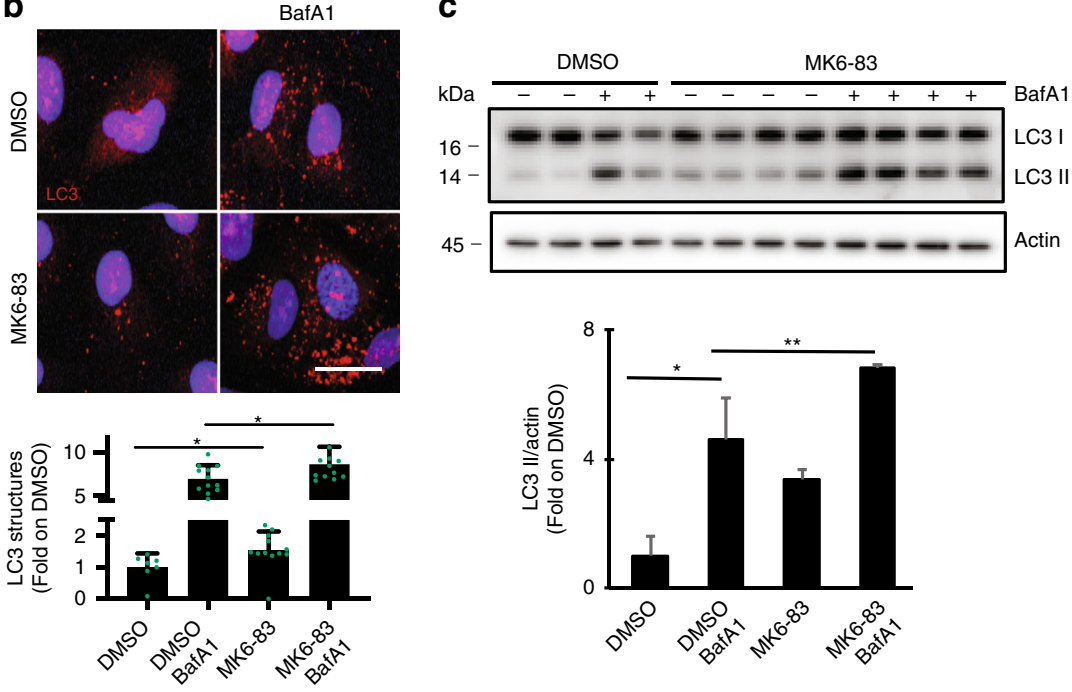

ML-SA1 -90 min
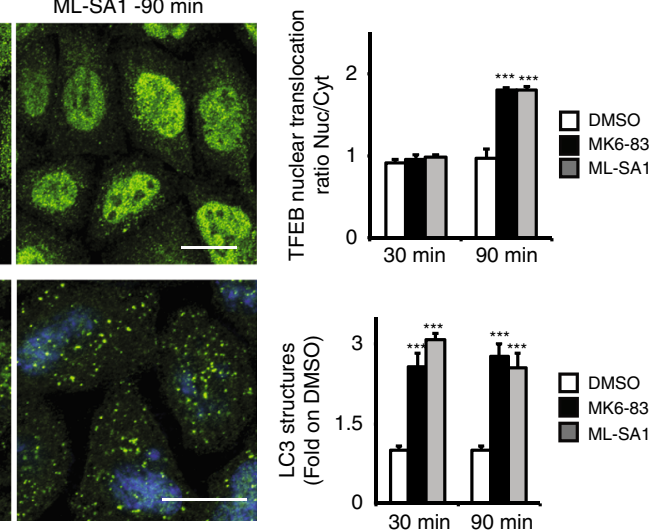

d
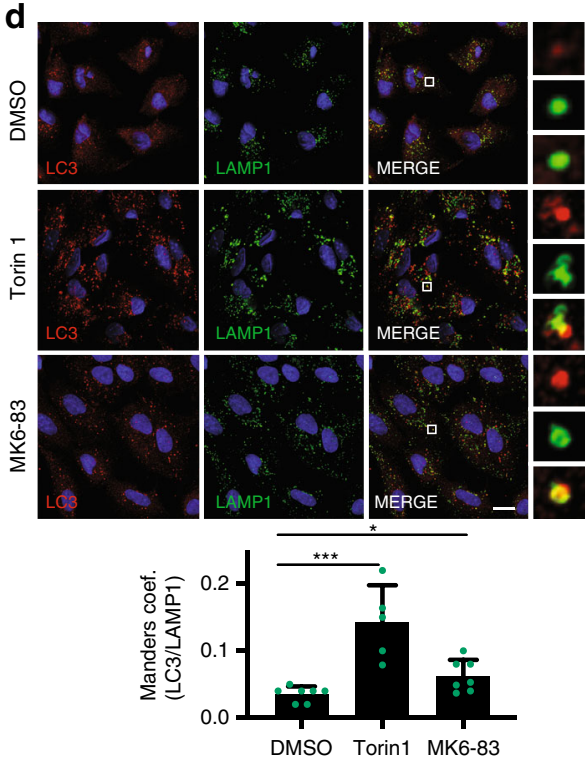

e

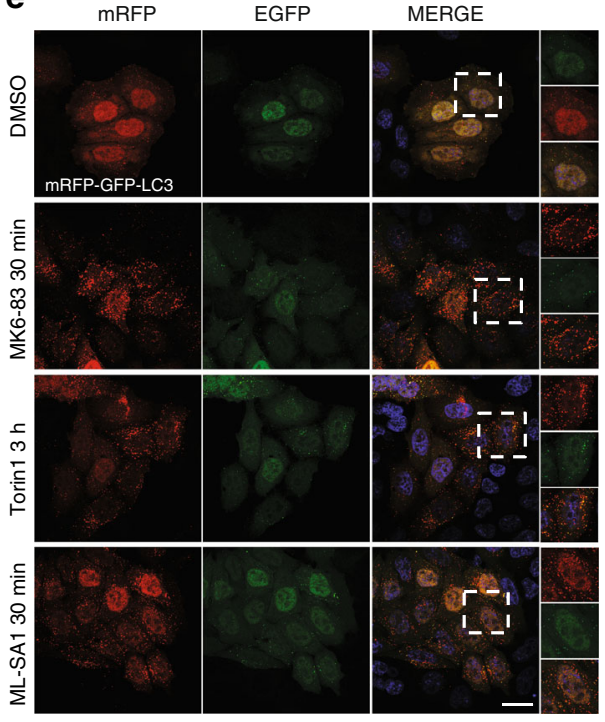

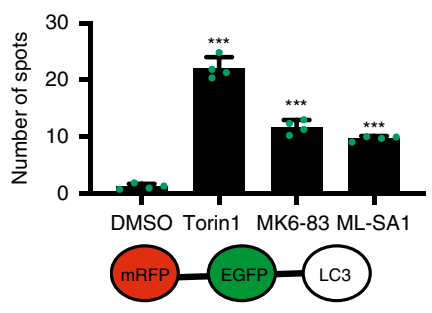

EGFP:autophagosome

mRFP:autolysosome+autophagosome f
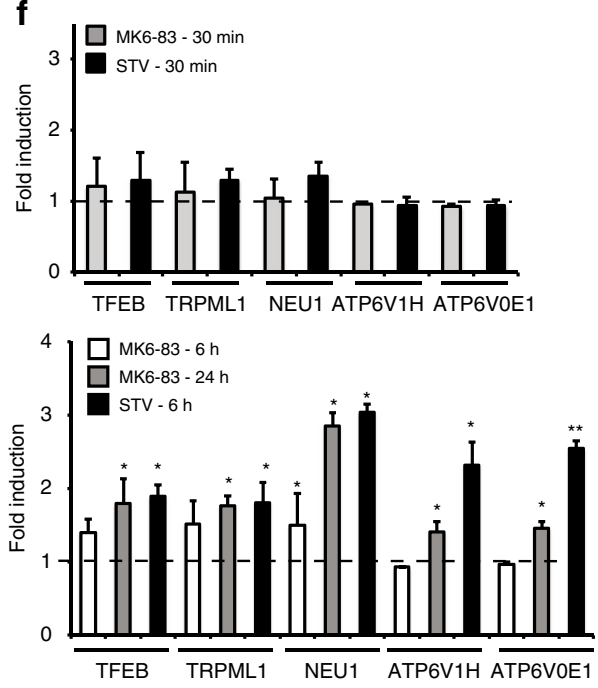

induces autophagosome formation and facilitates AV-lysosomal fusion.

To investigate the mechanism by which TRPML1 induces autophagosome formation, we examined the subcellular localization of TFEB, as TRPML1 was shown to induce TFEB nuclear translocation $^{23}$. Notably, $30 \mathrm{~min}$ after TRPML1 induction, a time when we observed a significant increase in the number of autophagosomes, TFEB was found in the cytoplasm (Fig. 1a) and the mRNA levels of a set of TFEB target genes were not increased (Fig. 1f), whereas at later time points of TRPML1 activation TFEB was detected in the nucleus (Fig. 1a) and the same set of mRNAs were upregulated (Fig. 1f). Similarly, co-treatment of MK6-83 
Fig. 1 Agonist-mediated activation of TRPML1 can induces autophagy in a TFEB-independent manner. a Representative confocal images of endogenous TFEB and LC3 localization in HeLa cells treated with DMSO, MK6-83, or ML-SA1 at different time points (30-90 min). The plot represents the TFEB nuclear to cytosol ratio and the quantification of the LC3 puncta per cell. Values are means \pm SD of $n=100$ cells pooled from three independent experiments. b Representative confocal images of endogenous LC3 in HeLa cells upon DMSO or MK6-83 treatment alone or in the presence of the lysosomal inhibitor bafilomycin A1 (BafA1). The plot shows LC3 puncta fold induction to DMSO-treated cells. Values are means \pm SD of $n=683$ cells pooled from three independent experiments. c Representative image of immunoblot analysis of endogenous LC3 (LC3I-II) upon MK6-83 treatment alone or in the presence of BafA1. Plot shows the densitometry of LC3II band normalized to Actin. The data in the graphs on the right are mean values \pm SD, $n=4$ lysates per condition pooled from four independent experiments. d Representative confocal images of endogenous LAMP1 and LC3 upon MK6-83, Torin1, or DMSO treatment in HeLa cells. Plot shows the Manders coefficient of LC3-LAMP1 colocalization. Values are means \pm SD of $n=215$ cells pooled from three independent experiments. e Representative images from high-content assay of HeLa cells stably overexpressing mRFP-EGFP-LC3 plasmid treated with DMSO, Torin1, MK6-83, or ML-SA1. Plot shows the quantification of the autolysosome number (spots negative for GFP and positive for RFP). f Quantitative PCR showing the mRNA levels of a subset of TFEB target genes upon MK6-83 treatment at different time points ( 30 min, 6 and $24 \mathrm{~h}$ ) and in control cells treated with HBSS. The data in the graphs are mean values $\pm S D, n=3$ samples per condition. Scale bar: $20 \mu \mathrm{m}$. $P$-values calculated by twotailed Student's $t$-test. ${ }^{\star} p$-value $<0.05 ;{ }^{\star \star} p$-value $<0.01 ;{ }^{\star \star \star} p$-value $<0.001$.

with the transcription inhibitor actinomycin D (ACT-D) still induced LC3 puncta formation, excluding a transcriptional effect during acute treatment (Supplementary Fig. 4b, c). Furthermore, acute MK6-83 treatment was able to induce LC3 puncta formation both in cells depleted of TFEB by small interfering RNAs (siRNAs) and, to a lesser extent, in TFEB/TFE3 double knockout $(\mathrm{KO})$ cells $^{36}$ (Supplementary Fig. 4d-f). In agreement with recent work ${ }^{37}$, the activation of TRPML1 by MK6-83 in normal nutrient conditions did not affect the phosphorylation of the mTORC1 substrates T389-PS6K and S6 ribosomal protein (Supplementary Fig. $4 \mathrm{~g}, \mathrm{~h}$ ), thus excluding a role of mammalian target of rapamycin (mTOR).

Together, these data indicate that acute TRPML1 activation is sufficient to induce autophagosome biogenesis in a TFEBindependent manner, suggesting that these effects are mediated by a different mechanism.

Activation of TRPML1 recruits PI3P-binding proteins to AVs. To identify the mechanism by which acute TRPML1 activation induces AV formation, we investigated the potential role of this lysosomal calcium channel in autophagy initiation. In mammalian cells, PI3P-enriched ER subdomains, known as omegasomes, act as platforms for AV formation upon autophagy induction ${ }^{38}$. To test whether TRPML1 is involved in phagophore formation, we acutely stimulated TRPML1 in HEK-293 cells that stably overexpress the omegasome marker double FYVE-containing protein 1 (DFCP1) fused to GFP (DFCP1-GFP) ${ }^{38}$. Cells treated with MK6-83 for $30 \mathrm{~min}$ showed a significant increase in the formation of DFCP1-GFP-puncta compared with vehicle-treated cells (Fig. 2a). The knockdown of TRPML1 decreased the induction of DFCP1-GFP puncta formation upon MK6-83 treatment confirming the selectivity of the agonist (Fig. 2b). Consistent with the results obtained with the TRPML1 activator MK6-83, overexpression of TRPML1 increased DFCP1-GFP puncta compared with the control vector (Fig. 2c). Moreover, we found that MK6-83-mediated activation ( $30 \mathrm{~min}$ ) of TRPML1 induced the recruitment of endogenous WD-repeat domain phosphoinositide-interacting WIPI2 puncta, an essential effector of the nascent $\mathrm{AV}^{39}$, in both HEK-293 and ARPE-19 cells (Fig. 2d). Similar results were obtained in ARPE-19 cells treated with ML-SA1 (Fig. 2e). WIPI2 is recruited to early PI3P-enriched autophagosomal structures along with ATG16L1 and is required for LC3 lipidation ${ }^{40}$. We found that upon TRPML1 activation, WIPI2 puncta colocalize with the endogenous ATG16L1 protein (Supplementary Fig. 5a), indicating that TRPML1 activation induces the formation of nascent phagophores that recruit the machinery of LC3 lipidation. Together, these data suggest that the direct activation of TRPML1 increases the recruitment of essential PI3P-binding proteins to the nascent AVs.
TRPML1-mediated generation of PI3P requires VPS34 and ULK1. The recruitment of DFCP1 and WIPI2 to the nascent AV requires the generation of $\mathrm{PI} 3 \mathrm{P}$ by the major autophagic protein complex hVPS34/class III phosphoinositide-3-kinase complex 1 $(\mathrm{PI} 3 \mathrm{KC} 3-\mathrm{C} 1)^{41}$. We found that specific PI3K inhibitors, such as wortmannin and the more selective compound SAR $405^{42}$, were able to fully block the effects of MK6-83-mediated activation of TRPML1 on WIPI2 puncta formation (30 min treatment; Fig. 3a). Similarly, siRNA-mediated silencing of VPS34 significantly reduced WIPI2 puncta in cells treated with MK6-83 (Fig. 3b). In agreement with the role of WIPI2 in the recruitment of LC3 to the phagophore ${ }^{40}$, we also found that pharmacological inhibition of VPS34 with SAR405 significantly reduced MK6-83-mediated induction of LC3 lipidation and LC3 puncta formation, as measured by immunoblot and immunofluorescence, respectively (Supplementary Fig. 5b, c). Thus, these results further suggest that TRPML1 can promote the formation and maturation of AVs.

Furthermore, both MK6-83 and ML-SA1 agonists were able to increase the production of PI3P, which was measured using a highly specific fluorescently labeled PX domain probe ${ }^{43,44}$, in a VPS34-dependent manner (Supplementary Fig. 5d). Most importantly, we also observed an elevation of immunoprecipitated VPS34 lipid kinase activity upon MK6-83 and ML-SA1 treatments (Supplementary Fig. 5e). The induction of autophagy requires the activation of ULK1 protein kinase complex, an event that is upstream of the activation of the VPS34 complex ${ }^{45}$. Thus, we tested whether MK6-83-mediated induction of WIPI2 puncta formation could be affected by the depletion of ULK1 complex activity. Silencing of $U L K 1 / 2$ fully inhibited WIPI2 puncta formation upon MK6-83 treatment (Fig. 3c and Supplementary Fig. $6 a, b, j, 1)$. Similar results were obtained using an inhibitor of ULK1 (MRT-68921) in two different cell lines (Fig. 3d). Finally, the ability of MK6-83 to induce WIPI2 puncta was significantly reduced in cells depleted of ATG13 (Fig. 3e and Supplementary Fig. 6e, 1) and in FIP200 KO mouse embryonic fibroblasts (MEFs) (Supplementary Fig. 7a), two critical components of the ULK1 complex, which are required for its assembly and for full ULK1 protein kinase activity ${ }^{46-49}$. Collectively, these results strongly indicate that activation of TRPML1 is sufficient to promote the generation of PI3P and AV biogenesis through activation of the two major protein complexes involved in autophagy initiation, ULK1, and VPS34.

TRPML1-mediated induction of AV formation requires CaMKK $\beta$. Intrigued by the link between TRPML1 activation and $\mathrm{AV}$ biogenesis, we investigated the molecular mechanisms activated downstream of the lysosomal calcium signaling. First, we asked whether TRPML1-mediated lysosomal calcium release is required for the recruitment of the autophagic PI3P-binding 
a

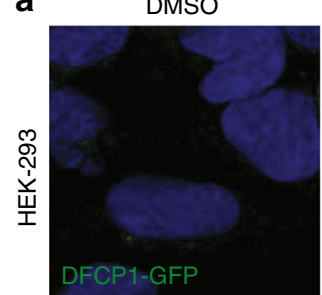

b

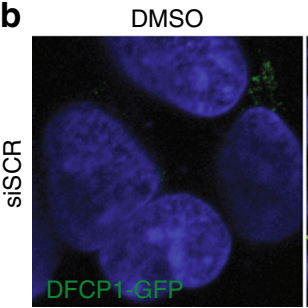

C

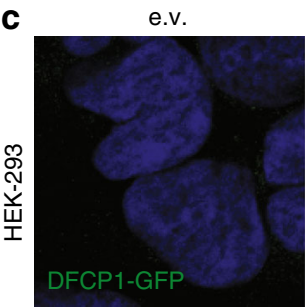

DMSO
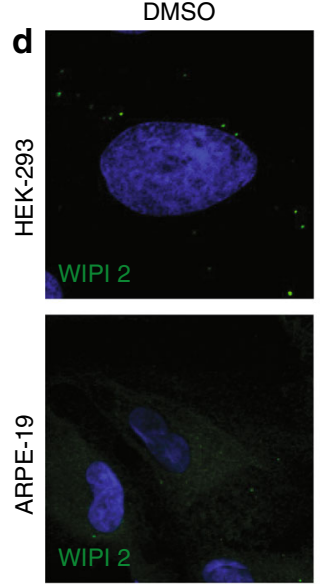

e

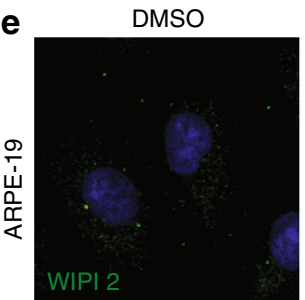

MK6-83

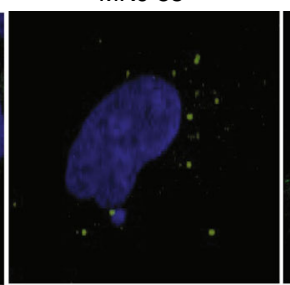

MK6-83

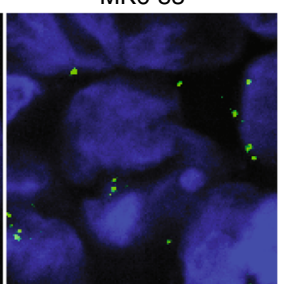

TRPML1

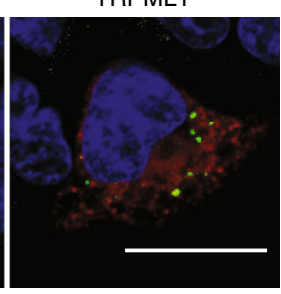

MK6-83
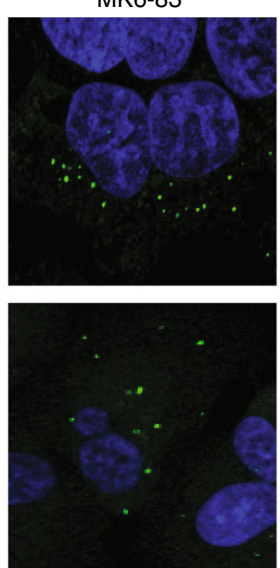

ML-SA1

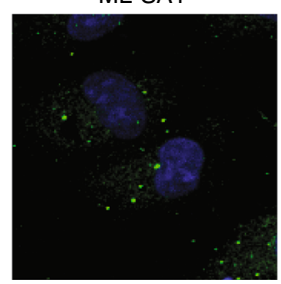

STV

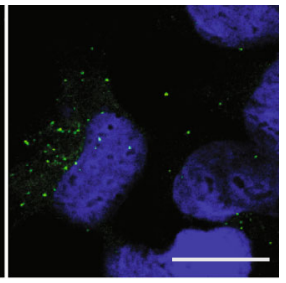

DMSO
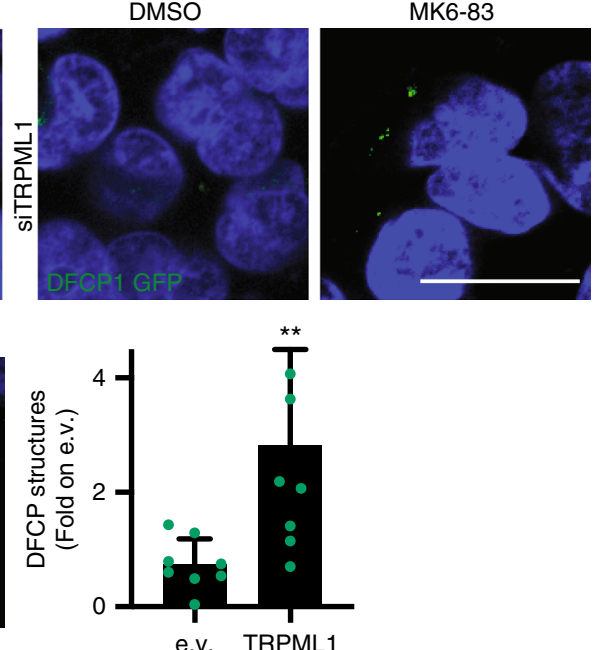
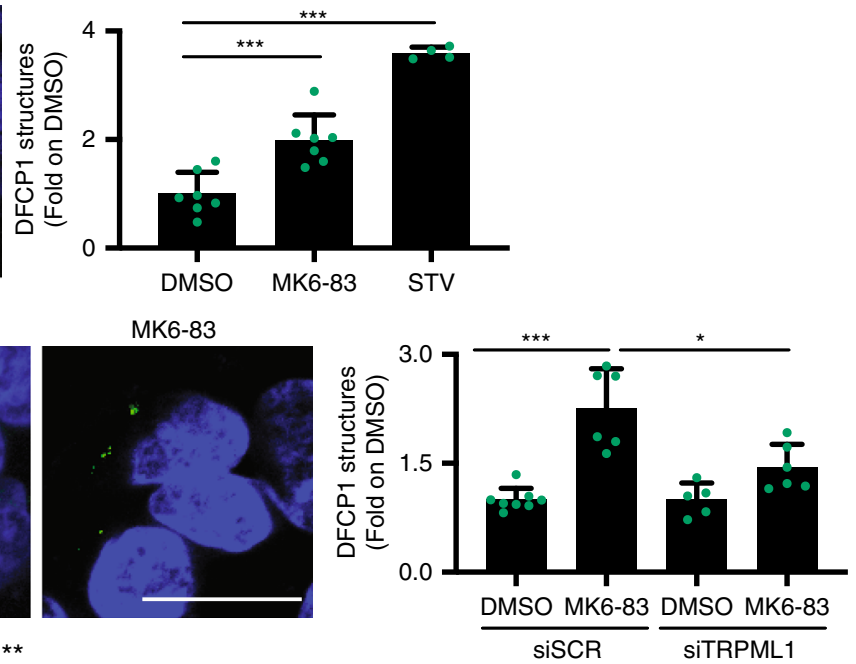

STV
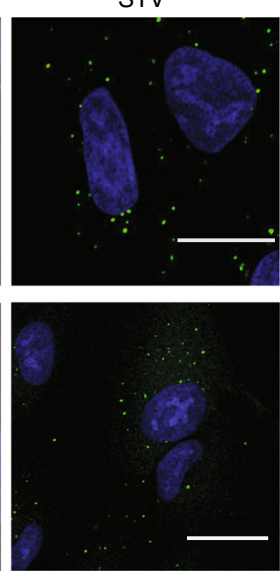

STV

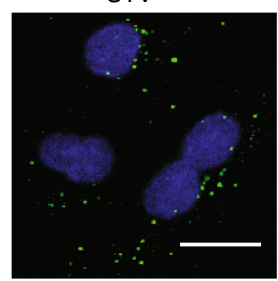

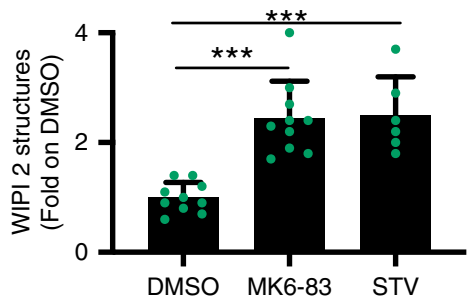

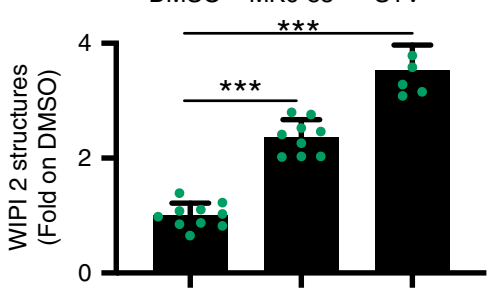

DMSO MK6-83 STV

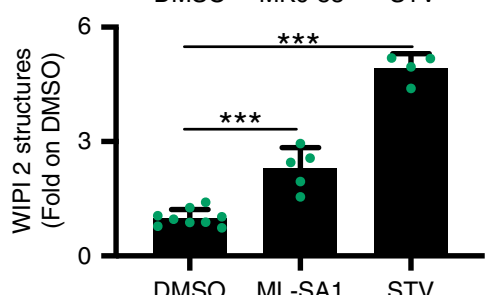

(Supplementary Fig. 7b). Most importantly, although the overexpression of GFP-tagged TRPML1, localizing in the lysosome, can induce WIPI2 puncta, the overexpression of a non-conducting pore mutant version of TRPML1 (TRPML1-DDKK ${ }^{50}$ ) does not (Supplementary Fig. 7c), confirming that the channel activity is required for phagophore formation.

The CaMKK $\beta$ is known to play a major role in the effects of intracellular $\mathrm{Ca}^{2+}$ on autophagy induction ${ }^{51}$. We found that the use of the CaMKK $\beta$ inhibitor STO- $609^{51}$ significantly reduced the formation of WIPI2 puncta in cells treated with MK6-83 (Fig. 4c). 
Fig. 2 TRPML1 activation increases the recruitment of the PI3P-binding proteins DFCP1 and WIPI2. a Representative confocal images of HEK-293 cells stably overexpressing DFCP1-GFP treated with DMSO, HBSS, or MK6-83. The plot shows the quantification of DFCP1 puncta formation as fold induction to DMSO-treated cells. Values are means \pm SD of $n>1000$ cells pooled from three independent experiments. b Representative confocal images of HEK-293 cells stably expressing DFCP1-GFP treated with a single siRNA molecule targeting TRPML1 (siTRPML1) or a scramble sequence (SCR) followed by treatment with MK6-83, DMSO, or HBSS. The plot shows the quantification of DFCP1 puncta formation as fold induction to DMSO-treated cells. Values are means \pm SD of $n>1000$ cells pooled from three independent experiments. c HEK-293 stably expressing DFCP1-GFP were transiently transfected with a vector encoding TRPML1 tagged with FLAG (in red) or an empty vector (e.v.). The plot shows the quantification of DFCP1 puncta as fold induction to the e. v.-transfected cells. Values are means \pm SD of $n=733$ cells pooled from three independent experiments. d Representative confocal images of endogenous WIPI2 in HEK-293 cells and in ARPE-19 cells upon treatment with MK6-83, DMSO, or STV (HBSS). Plots show the WIPI2 puncta quantification as fold induction to DMSO-treated cells. Values are means \pm SD of $n=167$ cells pooled from three independent experiments (HEK-293) and $n=320$ cells pooled from two independent experiments (ARPE-19). e Representative confocal images of ARPE-19 cells treated with ML-SA1, DMSO, or STV (HBSS). Plot shows WIPI2 quantification as fold induction to DMSO-treated cells. Values are means \pm SD of $n=722$ cells pooled from three independent experiments. $P$-values calculated by two tails Student's $t$-test. Scale bar: $20 \mu \mathrm{m}$. ${ }^{\star} p$-value $<0.05 ;{ }^{\star \star} p$-value $<0.01 ;{ }^{\star \star *} p$-value $<0.001$.

Consistently, silencing of CaMKK $\beta$ (Supplementary Fig. 6a, b, i, l) and its downstream effector calcium/calmodulin-dependent protein kinase I (CaMKI) (Supplementary Fig. 6a, b, i, l) significantly reduced WIPI2 puncta formation upon direct activation of TRPML1 with MK6-83 (Fig. 4d). Moreover, the depletion of CaMKI caused a reduction of LC3 lipidation upon MK6-83 treatment, as revealed by the co-treatment with bafilomycin A1 (Fig. 4e). The silencing of CaMKK $\beta$ or CaMKI did not affect TRPML1-mediated induction of TFEB nuclear translocation, indicating that these kinases are involved in TRPML1-dependent AV biogenesis but not in TRPML1dependent TFEB activation (Supplementary Fig. 7d). Thus, these results suggest the functional involvement of CaMKK $\beta / \mathrm{CaMKI}$ in regulating WIPI2 recruitment to the forming AV upon TRPML1 activation. Intracellular calcium levels can trigger autophagy by a CaMKK $\beta$-mediated activation of AMPK ${ }^{51-53}$. We observed that the AMPK inhibitor dorsomorphin (DM) ${ }^{51}$ resulted in a significant reduction of MK6-83-induced WIPI2 and DFCP1GFP puncta formation, in HeLa cells and HEK-293 cells, respectively (Fig. 4f, g). Moreover, the depletion of $A M P K$ by siRNAs (Supplementary Fig. 6a, b, l) significantly inhibits MK683 -induced WIPI2 puncta formation. Also, we found that MK683 was able to promote the activation of AMPK by elevating its phosphorylation on threonine 172 in WT fibroblasts but not in MLIV patient cells (Fig. 4h). Interestingly, AMPK induces autophagy by directly activating ULK1 via its phosphorylation on serine $555^{54,55}$. Consistently, MK6-83 treatment also enhances ULK1 S555 phosphorylation ${ }^{56}$. Conversely, co-treatment with STO-609 or DM completely abolished MK6-83-mediated activation of AMPK/ULK1 (Fig. 4i). In agreement with this result, overexpression of TRPML1 was able to enhance phosphorylation of ULK1 on serine 555 and Acetyl-CoA Carboxylase (ACC) on serine 79, both well-known direct AMPK substrates ${ }^{57}$ (Supplementary Fig. 7f). Interestingly, treatment with SN-2 in TRPML3overexpressing cells was able to induce the phosphorylation of the AMPK substrates ACC and ULK1, suggesting that in cells where TRPML3 is physiologically expressed at higher levels it may activate AV formation similar to TRPML1 (Supplementary Fig. $7 \mathrm{~g}$ ). Together, these results indicate that the induction of lysosomal TRPML1 activates the CaMKK $\beta /$ AMPK pathway to promote AV biogenesis through ULK1 activation.

TRPML1 activation promotes Beclin1 S15 phosphorylation. The autophagy protein Beclin1 is a central regulator of autophagy and functions through its interaction with the Class III PI3K VPS34, VPS15, and the autophagy protein ATG14 in the initial stages of AV formation ${ }^{58}$. We asked whether Beclin1 is necessary for TRPML1-mediated induction of AV formation. Interestingly, we found that the depletion of Beclin1 by siRNA-mediated silencing can significantly reduce TRPML1-mediated induction of
WIPI2 spots upon MK6-83 treatment (Fig. 5a and Supplementary Fig. $6 \mathrm{~g}, 1, \mathrm{~m})$.

Phosphorylation of Beclin1 on a specific serine (S15 in humans) by ULK1 is required for full autophagy induction through the activation of ATG14L-containing VPS34 complexes $^{59}$. We found that MK6-83-mediated activation of TRPML1 induces the phosphorylation of Beclin1 S15 as detected by using phospho-specific antibodies that recognize this phosphoserine in cells overexpressing WT pDS-RED-C1-Beclin1 (Fig. 5b). Next, we sought to test whether the MK6-83-mediated induction of the CaMKK $/$ /AMPK/ULK1 pathway mediates the phosphorylation of Beclin1 on S15. Therefore, Beclin1-overexpressing cells were stimulated with MK6-83 alone or in combination with inhibitors of CaMKK $\beta$ (STO-609), AMPK (DM), or ULK1 (MRT-68921). By following Beclin1 phosphorylation on S15, we found that these inhibitors were able to reduce the phosphorylation of Beclin1 (Fig. 5c), suggesting that MK6-83 can enhance VPS34 activity through the phosphorylation of Beclin1 on S15 via the CaMKK $\beta$ / AMPK/ULK1 pathway.

WIPI2 recruitment is reduced in cells from MLIV patients. TRPML1 mutations cause the human lysosomal storage disease mucolipidosis $\mathrm{IV}^{15,60}$. We investigated whether the induction of PI3P-binding protein recruitment was impaired in human fibroblasts derived from different MLIV patients (GM02526 and GM02527). In nutrient deprivation conditions such as Hank's balanced salt solution (HBSS) or amino acid starvation, WIPI2 puncta formation was significantly induced in WT fibroblasts but not in MLIV patient cells (Fig. 6a and Supplementary Fig. 8a). Similarly, starvation-mediated induction of WIPI2 puncta was reduced in ARPE-19 cells depleted of TRPML1 by genome editing (Supplementary Fig. 8b). Conversely, overexpression of WT TRPML1 was able to restore WIPI2 and autophagic induction in TRPML1-depleted cells (Supplementary Fig. 8c). A reduction of both DFCP1-GFP and WIPI2 puncta was observed by acute silencing of TRPML1 in stably DFCP1-GFP-transfected HEK-293 cells and ARPE-19 (Fig. 6b, c). Indeed, the induction of WIPI2 puncta was reduced in a MLIV cellular model generated by genome editing in human HAP-1 cells (Fig. 6d). Similar reduction of both WIPI2 (Fig. 6e) and LC3 (Supplementary Fig. 8d) puncta formation during starvation was also observed $1 \mathrm{~h}$ after treatment with the antagonist of TRPML1, ML-SI3 (Supplementary Fig. 9a). The inhibitory effects of ML-SI3 during starvation was observed also in cells silenced for TRPML3, indicating that TRPML3 does not play a major role in WIPI2 puncta formation during starvation (Supplementary Fig. 9b). Conversely, the activation of TRPML1 with MK6-83 has an additive effect elevating the formation of WIPI2 puncta during starvation (Supplementary Fig. 9c). We also confirmed a reduction of endogenous WIPI2 decorating phagophore-like structures and 


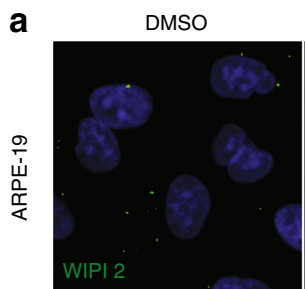

b
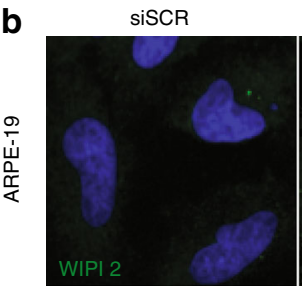

C

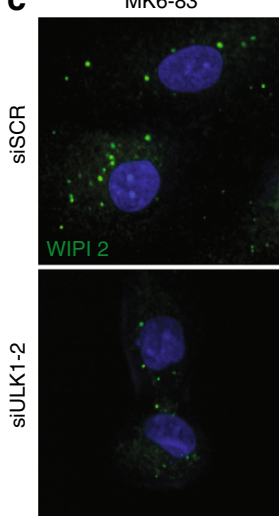

e

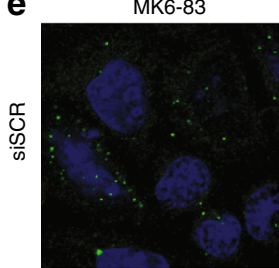

MK6-83

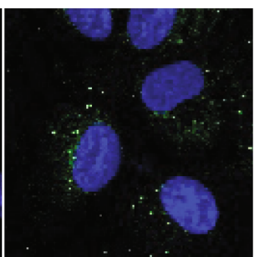

MK6-83

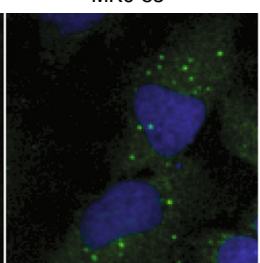

STV

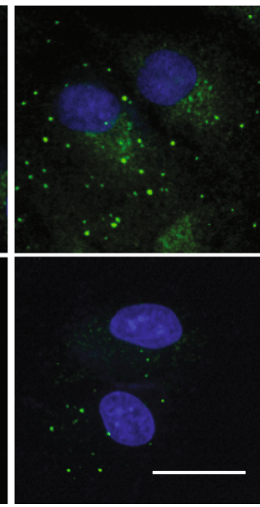

STV

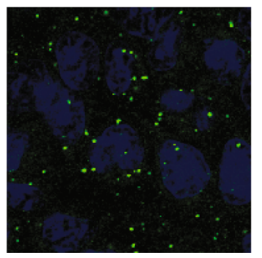

MK6-83+SAR 405

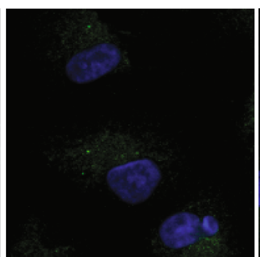

siVPS34
MK6-83+Wortmannin

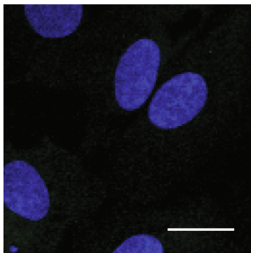

siVPS34 + MK6-83
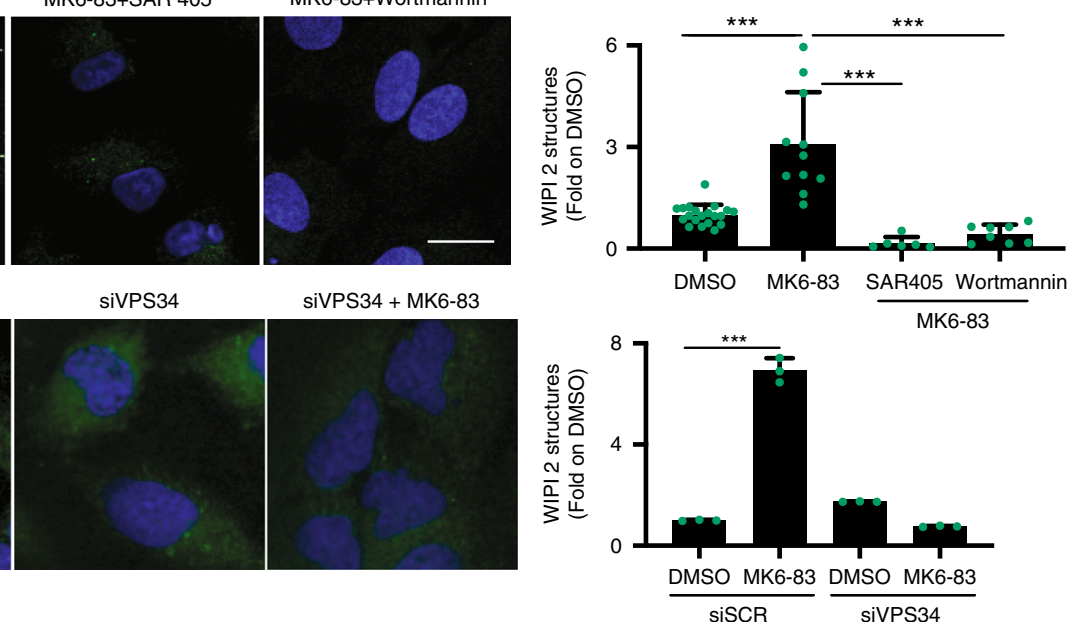

d MK6-83
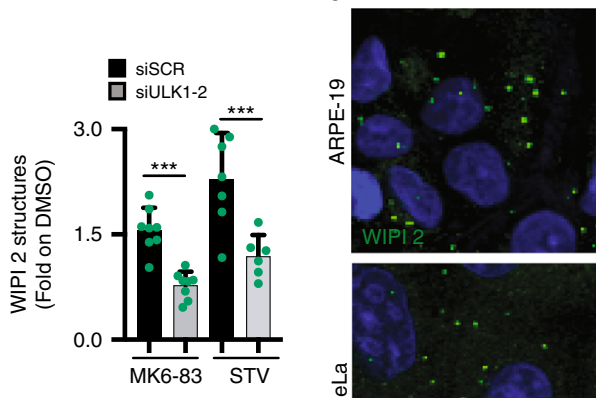

MK6-83 + MRT68921
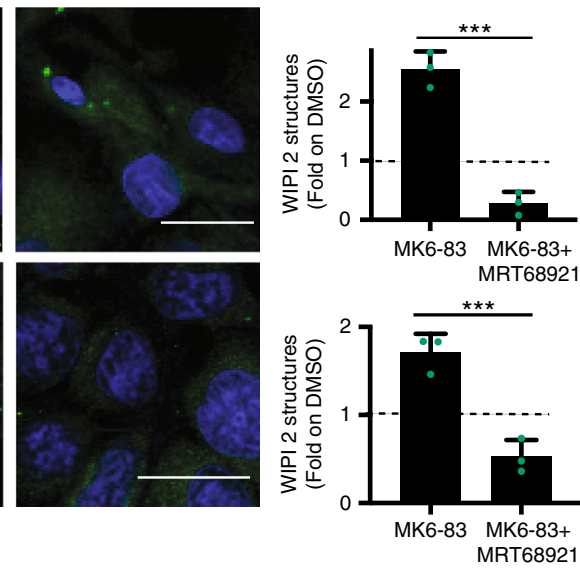

STV
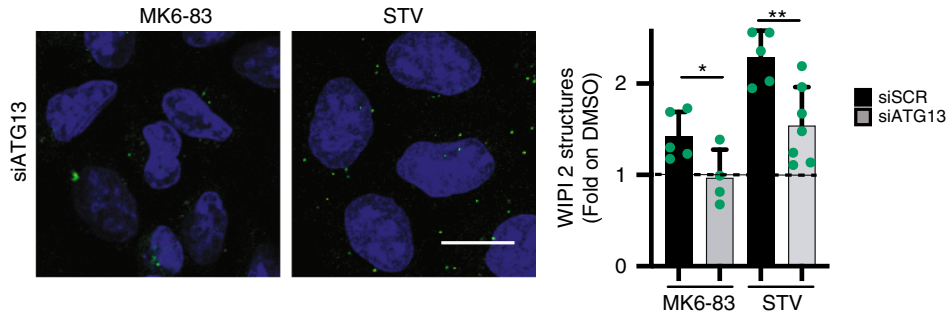

Fig. 3 PI3P generation through TRPML1 requires both hVPS34 and ULK1. a Representative confocal images of endogenous WIPI2 in ARPE-19 cells treated with DMSO and MK6-83 alone or in combination with SAR405 or wortmannin. Plot shows the quantification of WIPI2 puncta as fold induction to DMSO-treated cells. Values are means \pm SD of $n>1000$ cells pooled from three independent experiments. $\mathbf{b}$ Representative images from high-content assay of endogenous WIPI2 in ARPE-19 cells treated with a pool of three siRNA targeting VPS34 (siVPS34) or a scramble sequence. Values are means \pm SD of $n=200$ cells pooled from three independent experiments. c Representative images from cell-based high-content imaging assay of endogenous WIPI2 in ARPE-19 cells silenced with siRNA molecules targeting ULK1 and ULK2, followed by treatment with MK6-83 or STV (HBSS). Plot shows the quantification of WIPI2 puncta as fold induction to DMSO-treated cells. Values are means \pm SD of $n>1000$ cells pooled from three independent experiments. d Representative confocal images of endogenous WIPI2 in ARPE-19 and HeLa cells treated with MK6-83 alone or in combination with the ULK1 inhibitor (MRT-68921). Plots show the quantification of WIPI2 puncta as fold to DMSO. Values are means \pm SD of $n>1000$ cells pooled from four independent experiments. e Representative confocal images of endogenous WIPI2 in HeLa cells silenced with a pool of three siRNA molecules targeting ATG13 (siATG13) followed by treatment with MK6-83 or STV (HBSS). Plot shows the quantification of WIPI2 puncta as fold induction to DMSO-treated cells. Values are means \pm SD of $n>1000$ cells pooled from three independent experiments. $P$-values calculated by two tails Student's $t$-test. Scale bar: $20 \mu \mathrm{m}$. ${ }^{\star} p$-value $<0.05 ;{ }^{\star \star} p$-value $<0.01 ;{ }^{\star \star \star} p$-value $<0.001$.

associated ER membranes by immuno-electron microscopy analysis of starved human MLIV cells (Fig. $6 \mathrm{f})^{61}$. Notably, the reduction of WIPI2 puncta formation observed in MLIV cells was not due to the down-regulation of WIPI2 mRNA or protein levels, as quantitative PCR ( $\mathrm{PPCR}$ ) or immunoblot analysis in normal nutrient and starvation conditions resulted in similar WIPI2 content in both MLIV and WT fibroblasts (Supplementary
Fig. 10a, b). To further confirm that the reduction of PI3Pbinding proteins in TRPML1-depleted cells was due to a decrease in PI3P generation, we measured PI3P using the PX domain reporter in TRPML1-KO ARPE-19 cell line generated by genome editing. Indeed, we found a significant reduction of PX-positive puncta in KO cells compared to WT cells during autophagy induction (Supplementary Fig. 10c). 


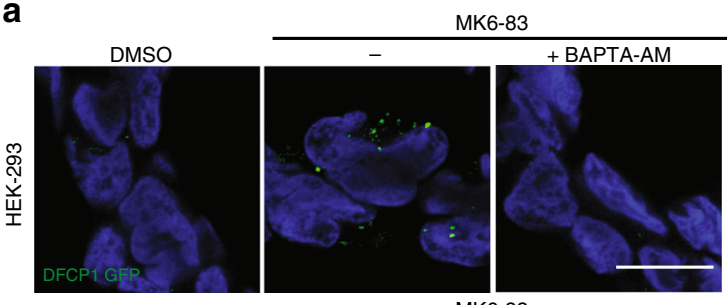

b

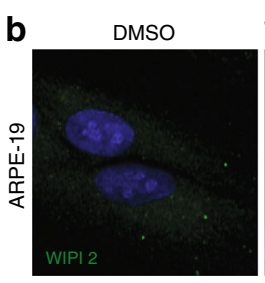

C

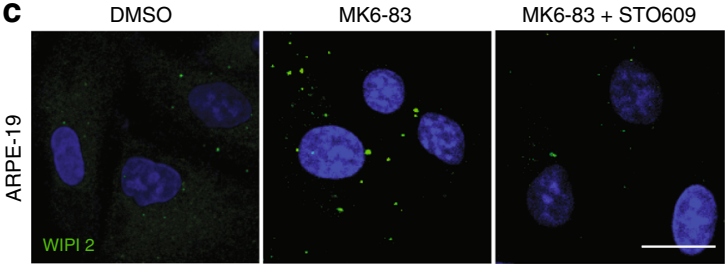

e
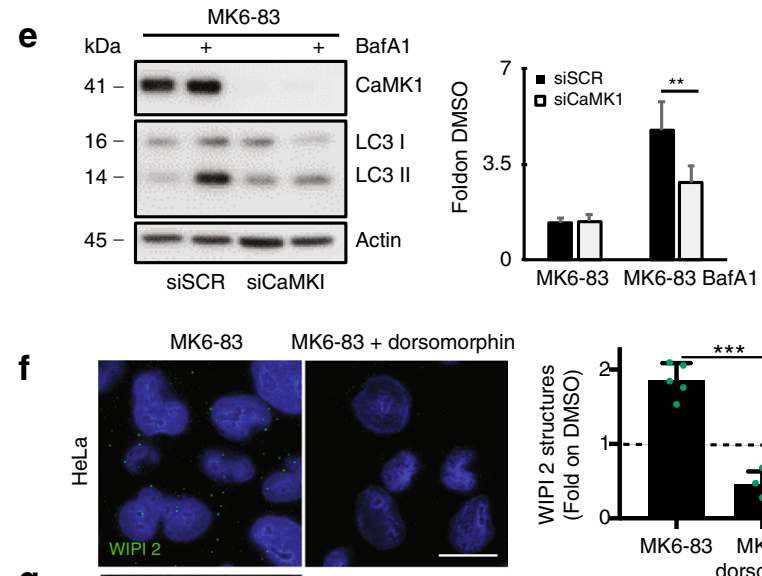

g
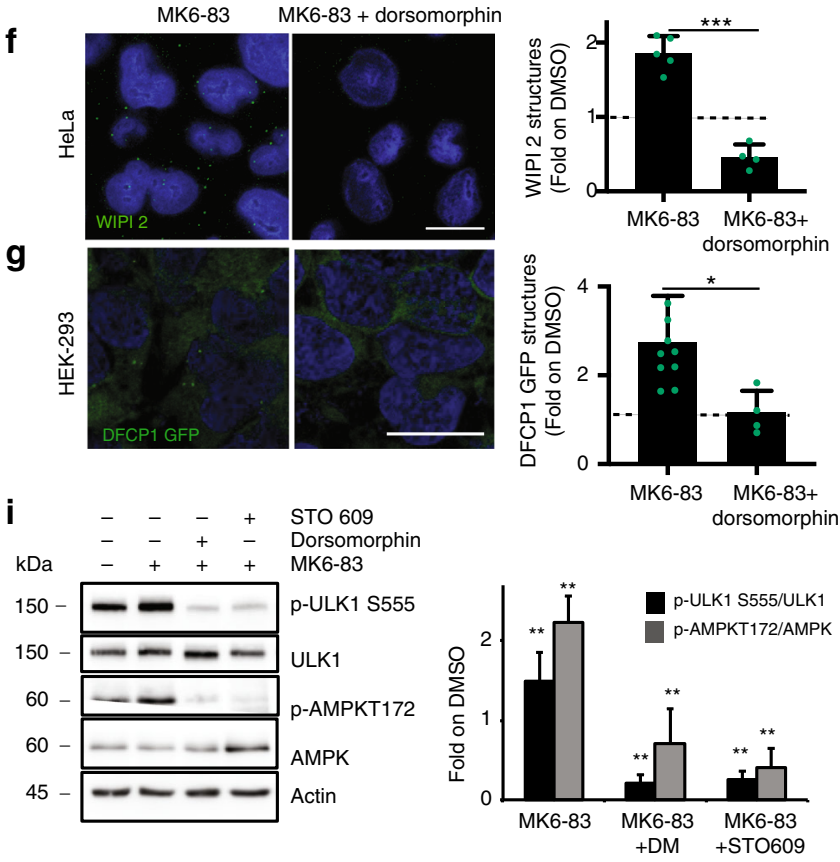

Altogether, our observations reveal a defective generation of PI3P and an impaired recruitment of essential PI3P-binding proteins to the nascent $\mathrm{AV}$ in various models of MLIV, suggesting that this may be a relevant mechanism in the disease pathogenesis.
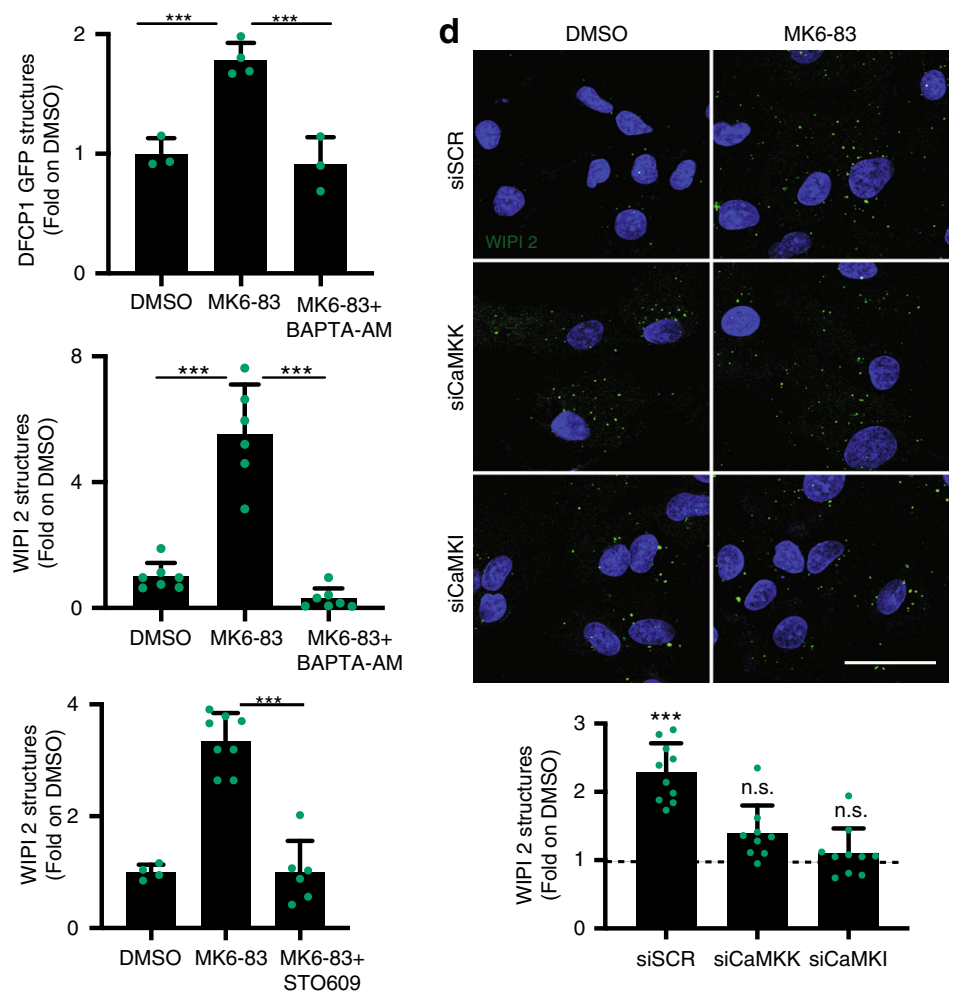

h
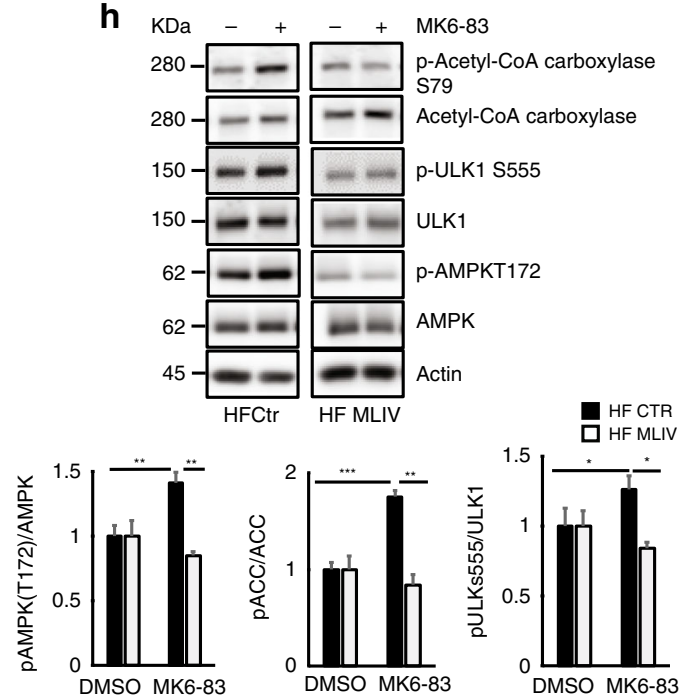

\section{Discussion}

Mammalian TRPML1 is a ubiquitously expressed non-selective, cation-permeable channel ${ }^{24}$, which mediates the release of $\mathrm{Ca}^{2+}$ from lysosomal or late endosomal lumen into the cytosol. TRPML1 is indispensable in the processes of endocytosis, 
Fig. 4 TRPML1-mediated lysosomal Ca2+ release induces AV biogenesis through CaMKK及. a Representative images of HEK-293 overexpressing DFCP1-GFP treated with DMSO and MK6-83 \pm BAPTA-AM. b Representative images of WIPI2 in ARPE-19 treated with DMSO and MK6-83 \pm BAPTA-AM. c Representative images of WIPI2 in ARPE-19 treated with DMSO and MK6-83 alone or in co-treatment with STO-609. d Representative images of WIPI2 in ARPE-19 treated with a pool of three siRNAs targeting CaMKK $\beta$ or CaMKI \pm MK6-83. e Representative image of immunoblot analysis of LC3 (LC3I-II) in HeLa cells silenced with siRNAs targeting CaMKI and treated with MK6-83 \pm bafilomycin A1 (BafA1). Plot shows the densitometry of LC3II normalized to actin as fold induction to DMSO. The data in the graphs on the right are mean values $\pm S D, n=4$ lysates per condition pooled from four independent experiments. $\mathbf{f}$ Representative images of WIPI2 in ARPE-19 treated with DMSO and MK6-83 \pm dorsomorphin. $\mathbf{g}$ Representative images of HEK-293 overexpressing DFCP1-GFP treated with DMSO and MK6-83 \pm dorsomorphin (DM). $\mathbf{h}$ Representative image of immunoblot analysis of phosphorylation AMPK on T172 and ULK1 on S555, Acetyl-CoA Carboxylase (ACC) on S79, upon MK6-83 treatment. Plot shows the densitometry of p-AMPK T172, p-ULK1 S555, and p-ACC S79 on AMPK, ULK1, and ACC normalized on Actin as fold induction to DMSO. The data in the graphs are mean values \pm SD, $n=$ 3 lysates per condition pooled from three independent experiments. i Representative image of immunoblot analysis of phosphorylation of AMPK on T172 and its substrate ULK1 on S555 upon MK6-83 treatment \pm STO-609 or dorsomorphin. Plot shows the densitometry of p-AMPK T172 and p-ULK1 S555 on AMPK and ULK1 normalized on actin as fold induction to DMSO. The data in the graphs are mean values \pm SD, $n=4$ lysates pooled from four independent experiments. a-d, $\mathbf{f}, \mathbf{g}$ Plot shows the quantification of -WIPI2 (b-d, $\mathbf{f})$, -DFCP $(\mathbf{a}, \mathbf{g})$ positive puncta as fold induction to DMSO-treated cells. Values are means \pm SD of $n=208(\mathbf{a}), n=225(\mathbf{b}), n=1169(\mathbf{c}), n=610(\mathbf{d}), n=385(\mathbf{f}), n=687$ (g) cells pooled from three independent experiments. Scale bar: $20 \mu \mathrm{m}$. ${ }^{\star} p$-value $<0.05 ;{ }^{\star \star} p$-value $<0.01 ;{ }^{* \star *} p$-value $<0.001$.

a

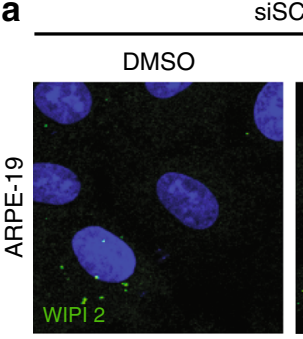
siSCR

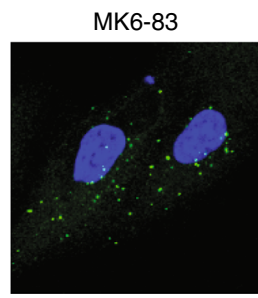

b

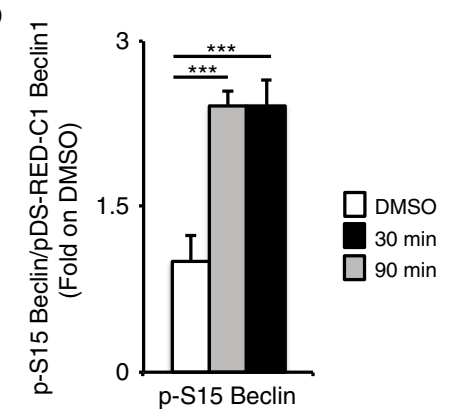

siBeclin1
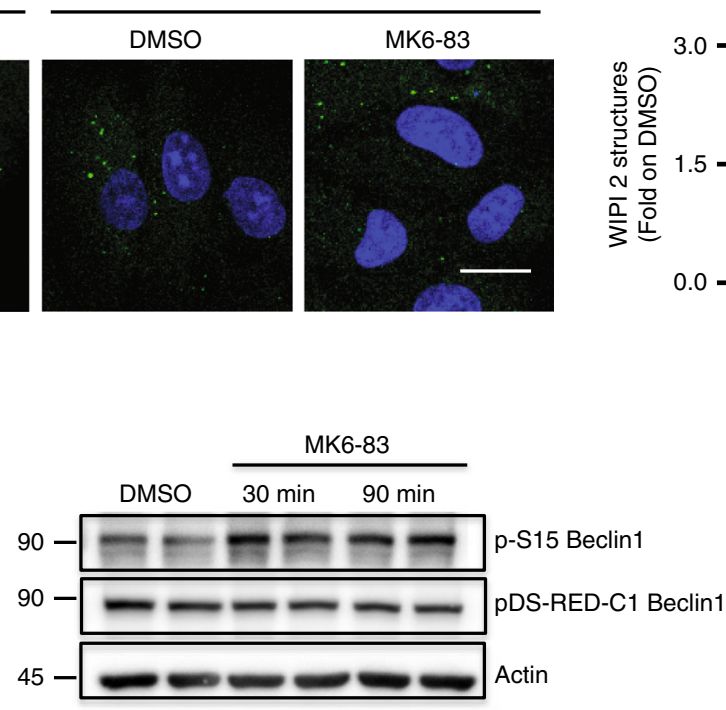

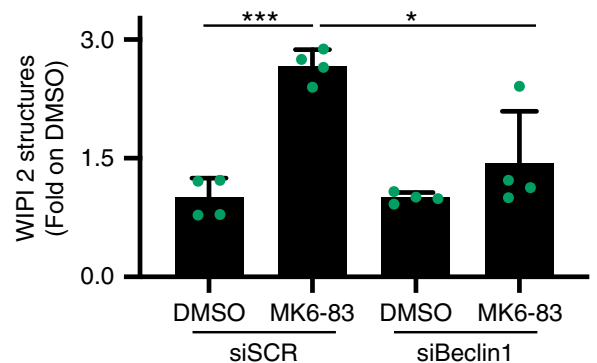

C
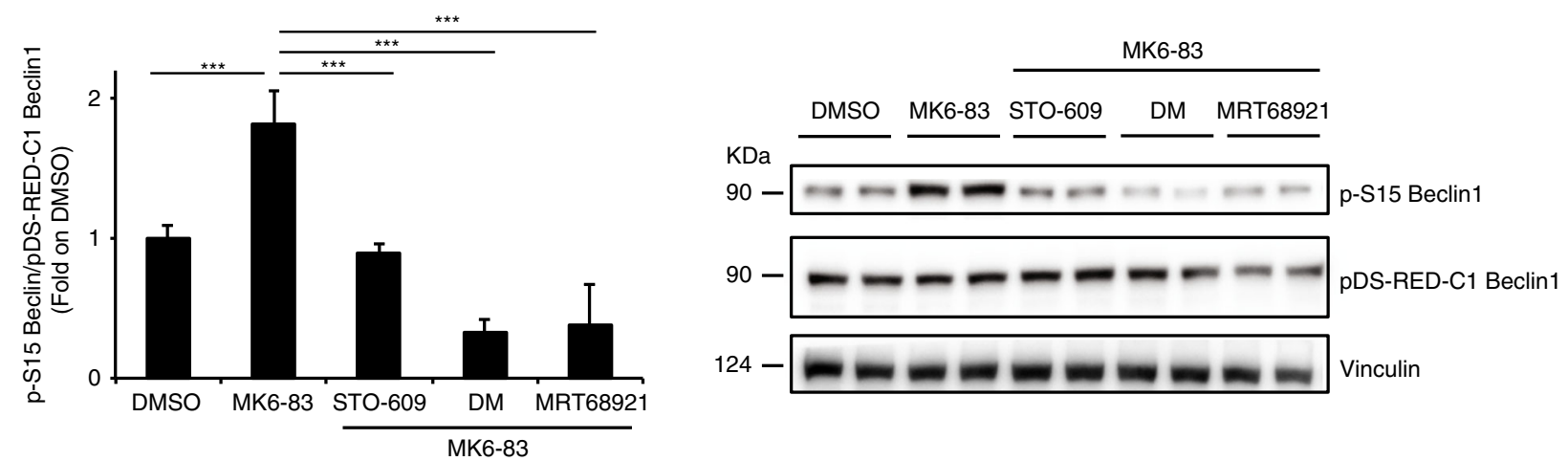

Fig. 5 Pharmacological activation of TRPML1 enhances Beclin1 S15 phosphorylation through the CaMKK/AMPK/ULK signaling pathway. a

Representative confocal images of endogenous WIPI2 in ARPE-19 cells treated with with a pool of three siRNA molecules targeting Beclin1 (siBeclin) followed by treatment with MK6-83. Plot shows the quantification of WIPI2-positive puncta as fold induction to DMSO-treated cells. Values are means \pm SD of $n=616$ cells pooled from three independent experiments. b Representative image of immunoblot analysis of phosphorylation of pDS-RED-C1-Beclin1 on serine 15 upon MK6-83 treatment at 30 and 90 min. Plot shows the densitometry of p-Beclin1 S15 on pDS-RED-C1-Beclin1 normalized on Vinculin as fold induction to DMSO-treated cells. The data in the graphs on the left are mean values $\pm S D, n=6$ lysates per condition pooled from three independent experiments. c Representative image of immunoblot analysis of phosphorylation of pDS-RED-C1-Beclin1 on serine 15 upon MK6-83 treatment alone or in combination with STO-609, dorsomorphin (DM), or MRT-68921. The data in the graphs on the left are mean values \pm SD, $n=6$ lysates per condition pooled from three independent experiments. $P$-values calculated by two tails Student's t-test. Scale bar: $20 \mu$ m. ${ }^{\star} p$-value $<0.05 ;{ }^{* \star} p$-value $<0.01$;

${ }^{\star \star \star} p$-value $<0.001$ 
a

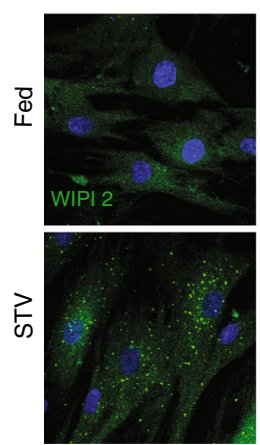

b
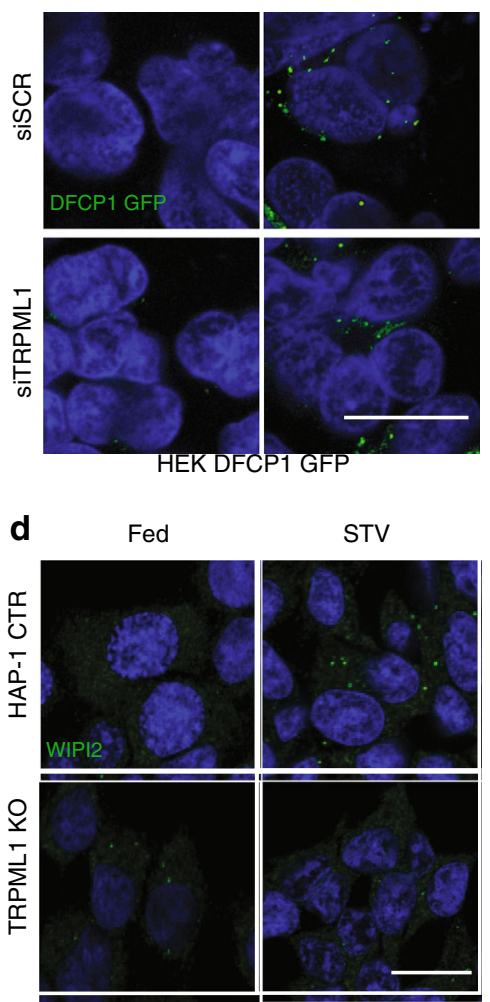

HF 2526

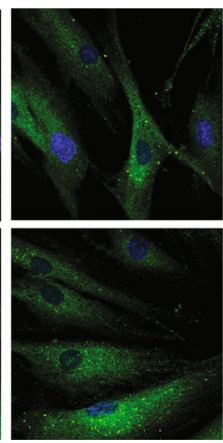

HF 2527

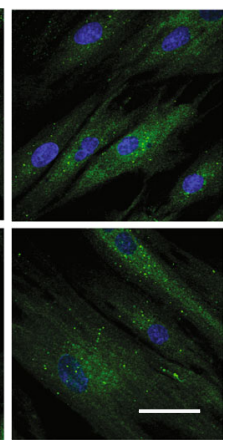

C Fed
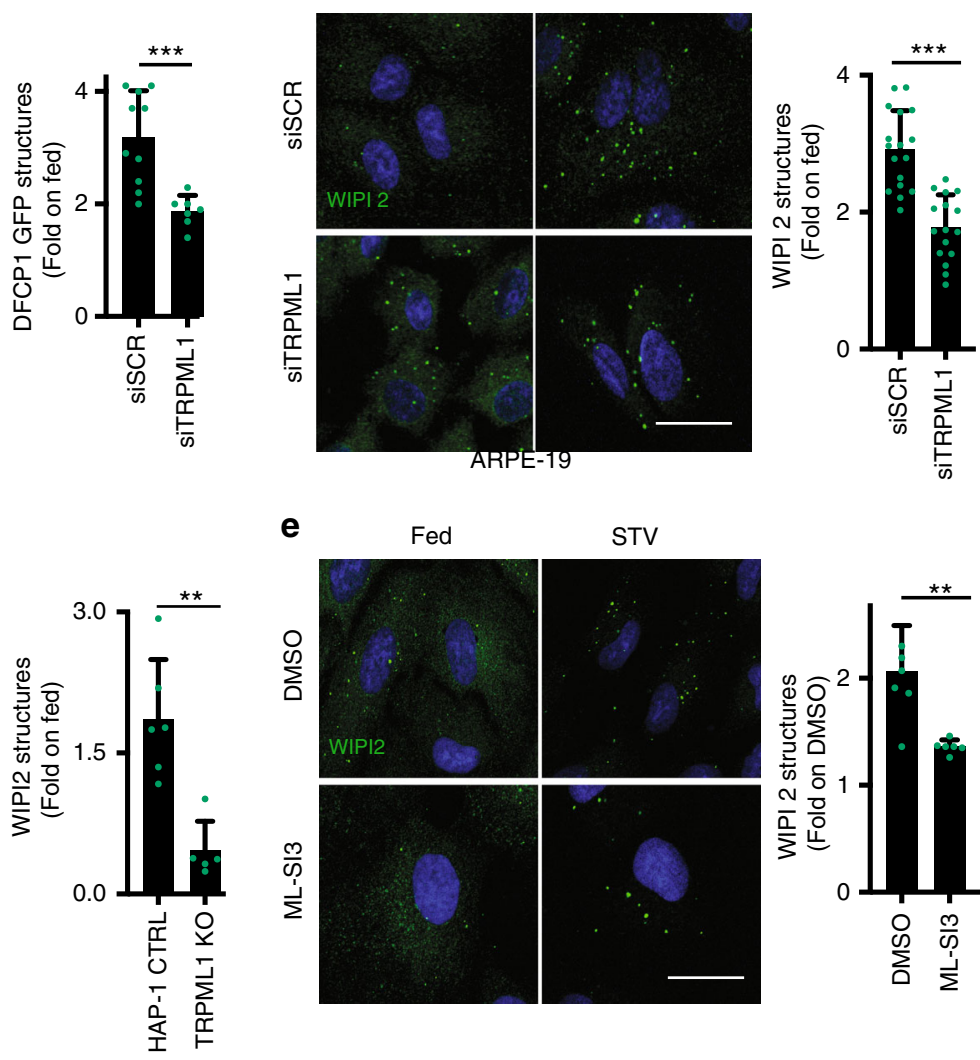

e

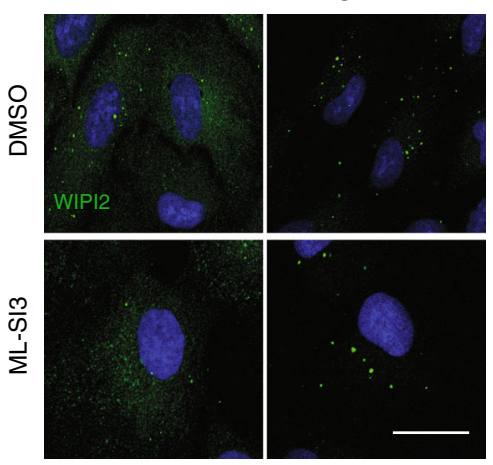

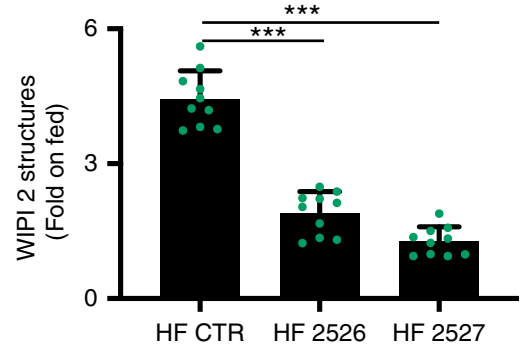

STV
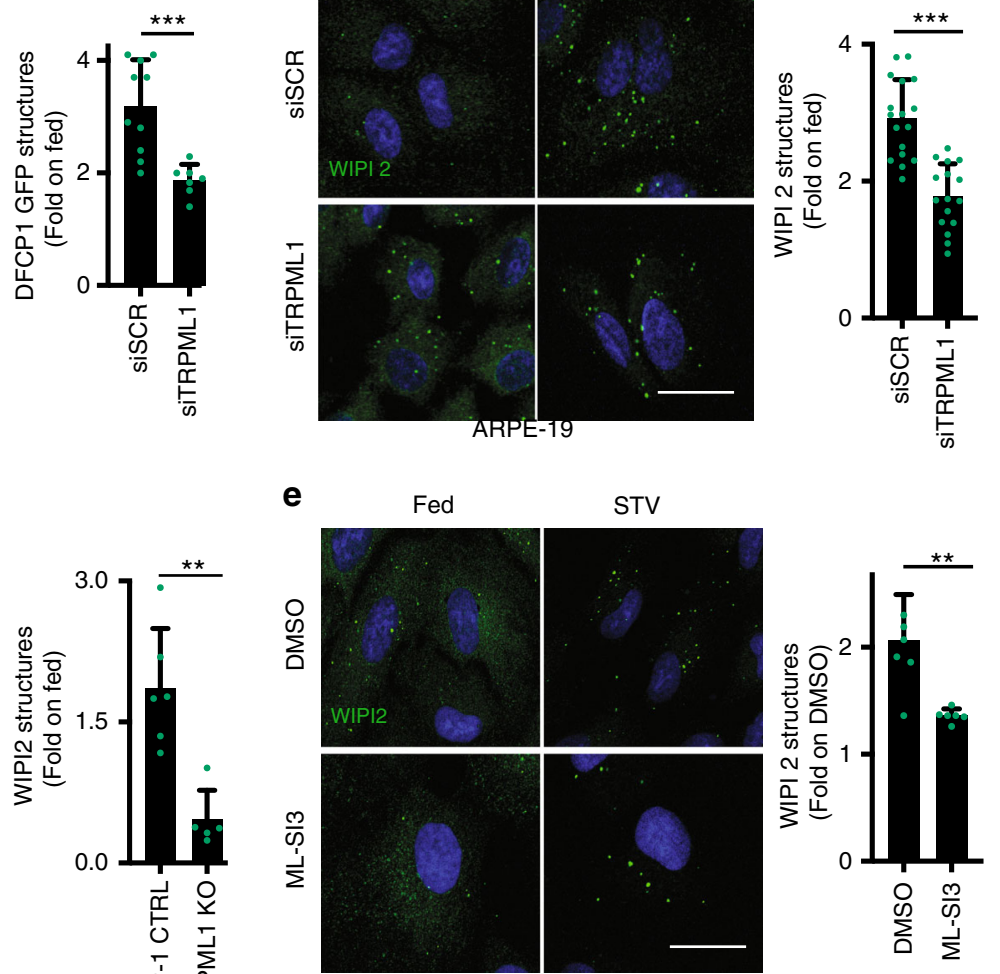

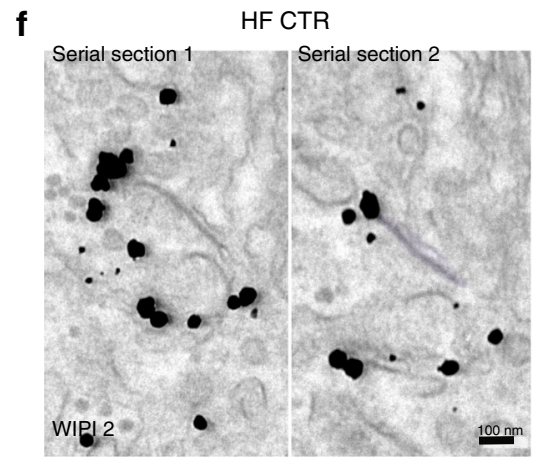

membrane trafficking, lysosomal exocytosis, and lysosomal biogenesis $^{23,28,29,62-65}$. Consistent with its role in lysosomal biogenesis, mutations in the TRPML1 gene cause the lysosomal storage disease MLIV, which is an autosomal recessive disorder characterized by delayed psychomotor development and vision impairment ${ }^{15-17}$. Notably, we showed that transcriptional activation of TRPML1 via TFEB promotes clearance of pathological

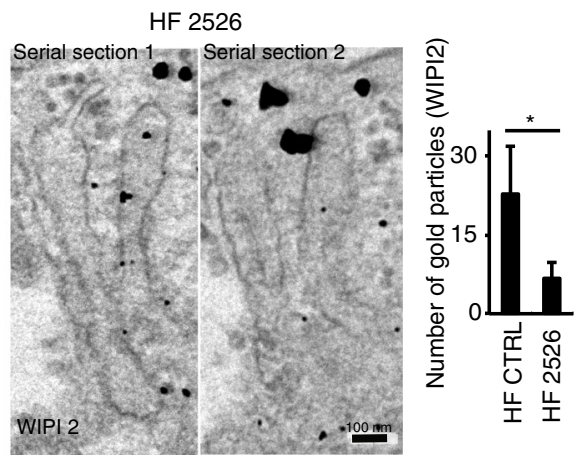

storage in a variety of LSDs through the activation of lysosomal exocytosis ${ }^{65}$.

Experimental- and structural-based studies indicate that TRPML1 is regulated by $\mathrm{pH}, \mathrm{Ca}^{2+}$, and phosphoinositides in a combined manner reflecting its ability to modulate highly dynamic trafficking processes such as endocytosis ${ }^{66-69}$. Interestingly, TRPML1 release activity can be modulated by nutrient 
Fig. 6 Induction of AV formation is reduced in Mucolipidosis type IV cells. a Representative confocal images of endogenous WIPI2 in human fibroblasts derived from a healthy individual (HF CTR) or from mucolipidosis type IV patient cell lines (GM02527, GM02526) incubated in complete medium or STV (HBSS). Plot shows the number of WIPI2 puncta per cell. Values are means \pm SD of $n=400$ total cells from three independent experiments. b Representative confocal images of HEK-293 cells overexpressing DFCP1-GFP treated with a single siRNA molecule targeting TRPML1 (siTRPML1) followed by treatment with complete medium (Fed) or STV (Starvation HBSS). Plot shows the quantification of DFCP1 positive puncta as fold induction to DMSO-treated cells. Values are means \pm SD of $n>1000$ cells pooled from three independent experiments. c Representative confocal images of endogenous WIPI2 in ARPE-19 cells treated with siRNA molecules targeting TRPML1 followed by incubation in complete medium or STV (Starvation HBSS). Plot shows the quantification of WIPI2-positive puncta as fold induction to DMSO-treated cells. Values are means \pm SD of $n=391$ cells pooled from three independent experiments. d Representative confocal images of endogenous WIPI2 in HAP-1 wild-type (CTR) or KO for TRPML1 incubated in complete medium or STV (HBSS). Plot shows the quantification of WIPI2 puncta as fold induction to DMSO-treated cells. Values are means \pm SD of $n=$ 925 cells pooled from three independent experiments. e Representative confocal images of endogenous WIPI2 in ARPE-19 cells, followed by treatment with the synthetic inhibitor of TRPML1 (ML-SI3). Plot shows the quantification of WIPI2 puncta as fold induction to DMSO-treated cells. Values are means \pm SD of $n=286$ cells pooled from three independent experiments. f TEM images of endogenous WIPI2 labeled by immunogold technique, in human fibroblasts derived from a healthy individual (HF CTRL) or derived from a mucolipidosis type IV patient (HF 2526). The plot shows the quantification of WIPI2 puncta. Values are means \pm SD of $n=726$ cells pooled from three independent experiments. $P$-values calculated by two-tailed Student's $t$-test. Scale bar: $20 \mu$ m, $\mathbf{f}$ : $100 \mathrm{~nm}$. ${ }^{\star} p$-value $<0.05 ;{ }^{\star \star} p$-value $<0.01 ;{ }^{\star \star \star} p$-value $<0.001$

deprivation activating a TFEB-driven transcriptional program through the induction of its nuclear translocation ${ }^{23}$. This mechanism may have an important role in the regulation of sustained autophagy during prolonged starvation conditions. However, although the complete process of transcription and subsequent translation of autophagic genes may require long kinetics, the acute activation of TRPML1-mediated lysosomal calcium mobilization may have consequences in the autophagy machinery at a shorter timescale. In the present study, we used synthetic agonists of TRPML1 to comprehensively investigate the effects of direct TRPML1 activation on autophagy. We found that acute pharmacological activation of TRPML1 rapidly induces AV formation and AV-lysosome fusion at early time points when TFEB is inactive being located predominantly in the cytosol. Our data are in agreement with previous work indicating that TRPML1 positively regulates membrane-trafficking processes such as vesicular fusion/fission events and autophagy $5,23,32,50,65,68,70-73$. There are also a few reports suggesting that TRPML1 modulation can block AV-lysosomal fusion ${ }^{74,75}$, although these observations might be explained by differences in the experimental conditions used and/or levels of TRPML1 overexpression. From a therapeutic perspective, it would be interesting to investigate whether excessive TRPML1 expression/ activation may impair both lysosomal ionic homeostasis and function promoting deleterious effects in specific cell types (e.g., cancer cells).

Our results indicate that acute TRPML1 activation induces AV formation independently of TFEB, suggesting that TRPML1 can modulate autophagy both in a rapid way, by promoting AV biogenesis and AV-lysosome fusion, and, in a sustained way, by inducing TFEB nuclear translocation and the transcription of autophagic and lysosomal genes, necessary to provide autophagic constituents during prolonged autophagy induction. Also, this mechanism is involved in a feedback loop by which TFEB positively regulates TRPML1 mRNA expression to further support long-term autophagy induction (working model Fig. 7) ${ }^{76}$.

In addition to TRPML1, the subfamily of TRPMLs includes TRPML2 and TRPML3. In contrast to TRPML1, these two channels show a very restricted tissue-specific expression and partially colocalize with late endosome/lysosomes ${ }^{24,30,62,77}$. By using cells depleted of each one of the channels, we found that TRPML1 activation is sufficient to induce AV biogenesis, also in the absence of TRPML2 or TRPML3. Then, by overexpressing TRPML2 and TRPML3, we confirmed that TRPML3, but not TRPML2, can induce LC3 puncta accumulation, as previously demonstrated $^{78-81}$. Similarly, the selective agonist of TRPML3, $\mathrm{SN}-2$, was able to weakly induce LC3 puncta formation in both
WT and TRPML1-depleted cells, indicating that TRPML3 may also modulate autophagy independently of the other channels, although most likely in tissues where it is physiologically more expressed ${ }^{20}$. Although a few articles have described that heterologously expressed TRPML protein subunits can interact with each other ${ }^{3,78,82}$, evidences are suggesting that endogenous TRPML subunits only partially colocalize and therefore exist mostly as homomeric channels ${ }^{82}$. Our observations are in agreement with these evidences, although further studies are necessary to fully understand whether endogenous TRPML3 activation can replace TRPML1-mediated autophagy effects. The answer to this question might be relevant for the development of therapeutics using selective TRPML3 agonists to revert the human MLIV phenotype.

In mammals, the initiation of phagophore formation is regulated by the sequential activation of the ATG1/ULK complex and the autophagy-specific PI3KC3-C1 (or VPS34) ${ }^{41,45}$. PI3KC3-C1 kinase activity generates PI3P at the pre-autophagosomal structure called omegasome ${ }^{38}$. Membrane-bound PI3P is recognized by proteins such as DFCP1 and WIPI proteins ${ }^{83}$. Here we found that the activation of TRPML1 triggers the recruitment of PI3Pbinding proteins DCFP1 and WIPI2, which are essential for nascent AVs. We also observed that upon TRPML1 activation, WIPI2-positive vesicles colocalize with ATG16L1, an essential component of the LC3 lipidation complex. WIPI2 is known to function downstream of PIK3C3/VPS34 and upstream of both the ATG12 and LC3 ubiquitin-like conjugation systems, and to trigger later steps of autophagosome generation ${ }^{40}$. Thus, our data suggest that TRPML1 activation and lysosomal calcium may also play a role in AV maturation through the induction of LC3 lipidation. Consistent with this hypothesis, we also found that TRPML1 activation increases AV-lysosomal fusion, a process that can be pharmacologically inhibited by vinblastine or by silencing of the autophagic SNARE syntaxin-17. Intriguingly, we observed that in addition to promoting autophagosome formation and autophagosome-lysosome fusion, TRPML1 activation also induces LC3 lipidation of endolysosomal structures, an effect that was previously observed in cells treated with other autophagy modulators $^{35}$.

The recruitment of DFCP1 and WIPI2, upon TRPML1 activation, is likely a consequence of the elevation of PI3P levels, which requires both ULK1 and VPS34 activity, two essential protein complexes involved in the first steps of autophagy initiation $^{38}$. Interestingly, we found defects in the induction of PI3P-binding protein recruitment to phagophore in fibroblasts from MLIV patients who carry loss-of-function mutations in the TRPML1 gene. These results indicate that TRPML1 activity can 


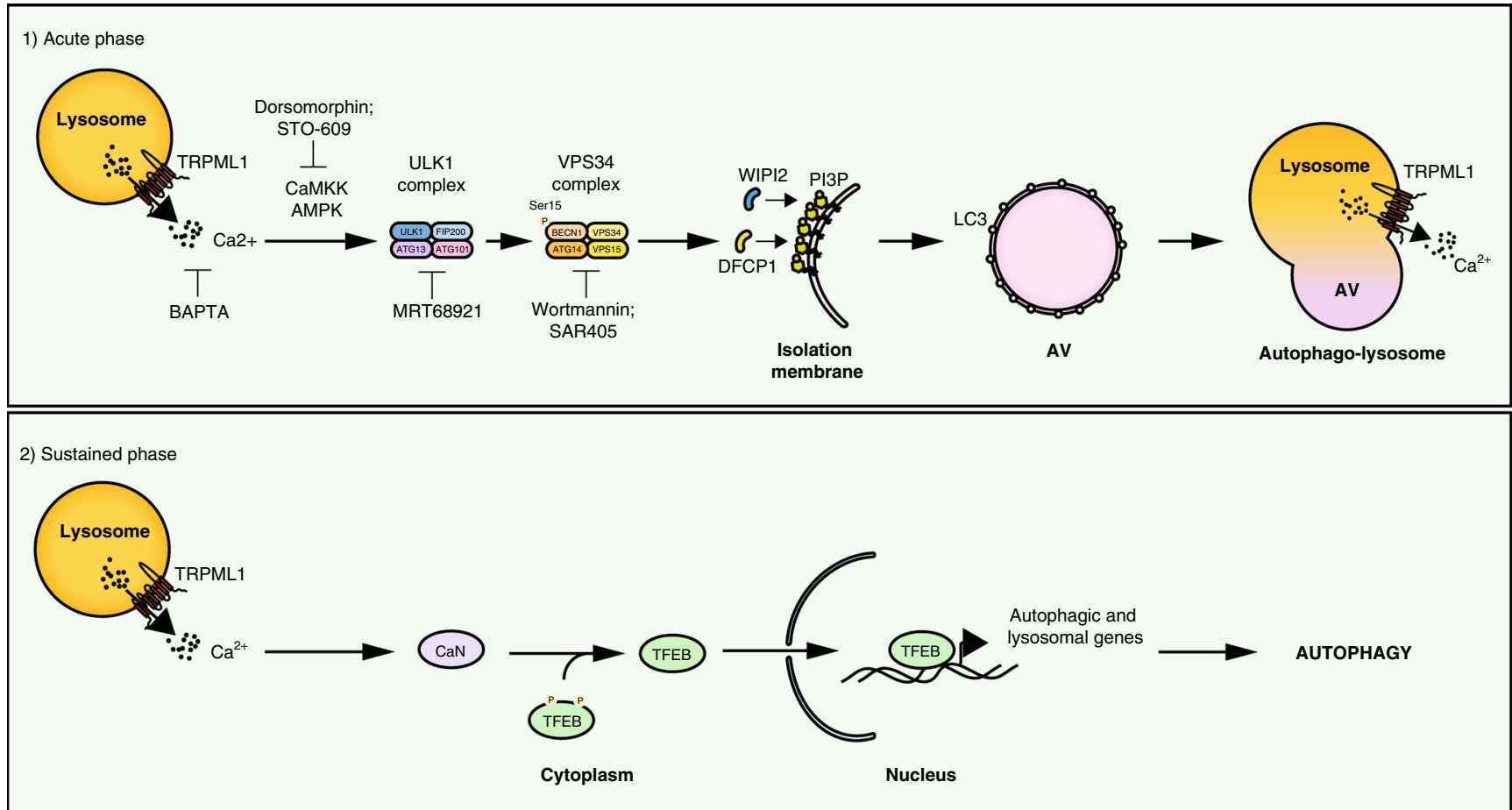

Fig. 7 Model of TRPML1 regulation of autophagy. Model of multistep regulation of autophagy by TRPML1. TRPML1 modulates autophagy in two steps: (1) by rapidly inducing $\mathrm{AV}$ biogenesis and AV-lysosomal fusion through the activation of the calcium/calmodulin-dependent protein kinase kinase (CaMKK $\beta$ )mediated signaling and the two major protein complexes involved in autophagy initiation, ULK1 and hVPS34, and (2) by inducing TFEB nuclear translocation that promotes the transcription of autophagic and lysosomal genes, which is necessary for a sustained induction of autophagy.

activate the ULK1 complex and subsequently VPS34, and suggest that the impairment of this pathway is a relevant mechanism in MLIV pathogenesis.

The role of intracellular calcium levels during phagophore formation as well as the downstream pathways involved in this process have not been fully elucidated. Previous studies demonstrated that binding of PIK3C3/VPS34 to calmodulin and calcium is required for the activity of VPS34 during bacterial infection $^{84,85}$. CaMKK $\beta$ can activate autophagy through both AMPKdependent $^{51-53}$ and -independent mechanisms ${ }^{86}$. Also, rapid AMPK activation during autophagy initiation was described during acute $(<30 \mathrm{~min})$ starvation. Interestingly, this acute response involves modulation of AMPK/ULK1 complexes, whereas it does not affect the more abundant ULK1/ATG13 complex ${ }^{87}$. Whether lysosomal calcium can promote autophagosome biogenesis through these pathways is completely unknown. Here we found that TRPML1-mediated signaling requires the activation of CaMKK $\beta$ and its substrate AMPK, a major nutrient-sensing kinase that regulates autophagy through phosphorylation of both ULK1 and Beclin1, and the subsequent induction of the VPS34 complex ${ }^{54,88,89}$. Thus, although acute TRPML1 activation may induce AV formation through CaMKK $\beta / A M P K$ pathway, prolonged starvation can induce autophagy through TFEB. Interestingly, TRPML1 and TFEB pathways have been also involved in mTORC1 reactivation during prolonged starvation ${ }^{74,90}$, a mechanism that may limit the deleterious effects of excessive autophagy.

Overall, our data, together with previously published observations, indicate that the physiological role of TRPML1 during autophagy is more complex than expected ${ }^{23}$. Thus, our results suggest that TRPML1 can use multiple pathways to modulate autophagy and lysosomal biogenesis, indicating that this channel is an important hub for cell metabolism and a promising therapeutic target for a variety of disease conditions.

\section{Methods}

Drugs and cellular treatments. The following drugs were used to perform the assays: Torin $1(1 \mu \mathrm{M}$, Tocris Bioscience, $3 \mathrm{~h})$; BAPTA-AM $(10 \mu \mathrm{M}$, Thermo Fisher, cell was pre-treated for $30 \mathrm{~min}$ and then used in co-treatment with MK-683 for additional $30 \mathrm{~min})$; EGTA-AM $(10 \mu \mathrm{M}$, Thermo Fisher, cell was pre-treated for $30 \mathrm{~min}$ and then used in co-treatment with MK-683 for additional $30 \mathrm{~min}$ ); MK6$83(30 \mu \mathrm{M}$ for $30 \mathrm{~min}$ or otherwise indicated in the text, Tocris or Dr C. Grimm from Ludwig Maximilian University); ML-SA1 $(30 \mu \mathrm{M}$, Sigma, $30 \mathrm{~min}$ or otherwise indicated in the text); ML2-SA1/EVP22 (30 $\mu \mathrm{M}$, gift from Professor Grimm C. from Ludwig Maximillian University of Munich, $30 \mathrm{~min}$ or otherwise indicated in the text); SN-2 (30 $\mu \mathrm{M}$, gift from Professor Grimm C. from Ludwig Maximillian University of Munich, $30 \mathrm{~min}$ or otherwise indicated in the text); Bafilomycin Al $(100 \mathrm{nM}$, Sigma, $3 \mathrm{~h})$; STO-609 (20 $\mathrm{MM}$, Sigma, cells were pre-treated for $30 \mathrm{~min}$ and then used in co-treatment with MK-683 for additional $30 \mathrm{~min})$; wortmannin ( $5 \mu \mathrm{M}$, Sigma, $30 \mathrm{~min})$; SAR405 (0.5 $\mu \mathrm{M}$, ApexBio, $30 \mathrm{~min})$; DM ( $40 \mu \mathrm{M}$, Tocris, $30 \mathrm{~min})$; MRT-68921 (30 $\mathrm{MM}$, Tocris Bioscience, cells were pre-treated for $30 \mathrm{~min}$ and then used in co-treatment with MK-683 for additional $30 \mathrm{~min}$ ) ACT-D $\left(0.1 \mu \mathrm{g} \mathrm{mL}^{-1}\right.$, Sigma, $\left.3 \mathrm{~h}\right)$; Vinblastine $(20 \mu \mathrm{M}$, Sigma-Aldrich, cells were pretreated for $2.5 \mathrm{~h}$ and then used in co-treatment with MK-683 for additional $30 \mathrm{~min}$ ). The starvation medium was prepared with HBSS (Thermo fisher), $10 \mathrm{mM}$ HEPES (Sigma), for amino acid starvation medium we used RPMI without amino acid from US Biological (R9010-01) supplemented with 10\% dialyzed fetal bovine serum (Thermo fisher).

Cell culture and transfection. ARPE-19 (retinal pigment epithelium cell line), HEK-293, and HeLa M cells were purchased from American Type Culture Collection (ATCC) and were cultured in Dulbecco's modified Eagle's medium (DMEM) F12 and DMEM, respectively, supplemented with $10 \%$ fetal bovine serum, $200 \mu \mathrm{M}$ L-glutamine, $100 \mu \mathrm{M}$ sodium pyruvate, $5 \% \mathrm{CO}_{2}$ at $37^{\circ} \mathrm{C}$. In addition to other cell lines, ARPE-19 cells were chosen to add a diploid nontransformed human cell line to confirm our data. ARPE-19 TRPML1-KO cells were generated by Dr J. Monfregola at TIGEM (Naples); ARPE-19 TRPML2 KO cell was generated by Dr A. Amabile at San Raffaele Institute (Milan); both of them were cultured in DMEM F12 supplemented with $10 \%$ fetal bovine serum, $200 \mu \mathrm{M}$ L-glutamine, $100 \mu \mathrm{M}$ sodium pyruvate, $5 \% \mathrm{CO}_{2}$ at $37^{\circ} \mathrm{C}$.

HAP-1 (TRPML1-KO, TRPML3-KO, and Ctr) cells were purchased from Horizon and were cultured in IMDM supplemented with $10 \%$ fetal bovine serum, $200 \mu \mathrm{M}$ L-glutamine, $100 \mu \mathrm{M}$ sodium pyruvate, $5 \% \mathrm{CO}_{2}$ at $37^{\circ} \mathrm{C}$. Control (CTR) human patient fibroblasts were provided by Dr N. Brunetti (TIGEM); GM02526 and GM02527 were purchased from Coriell Institute and were cultured in DMEM supplemented with $15 \%$ fetal bovine serum, $200 \mu \mathrm{M}$ L-glutamine, $5 \% \mathrm{CO}_{2}$ at $37^{\circ} \mathrm{C}$. 
Hek-293 DFCP GFP was a gift of Dr Ktistakis and were cultured in DMEM supplemented with $10 \%$ fetal bovine serum, $200 \mu \mathrm{M}$ L-glutamine, $100 \mu \mathrm{M}$ sodium pyruvate, $0.8 \mathrm{mg} \mathrm{mL}^{-1}$ of G418, $5 \% \mathrm{CO}_{2}$ at $37^{\circ} \mathrm{C}$. MEF WT and MEF FIP200 KO were a gift of Dr C. Settembre from TIGEM (Naples) and were cultured in DMEM supplemented with $10 \%$ fetal bovine serum, $200 \mu \mathrm{M}$ L-glutamine, $5 \% \mathrm{CO}_{2}$ at $37^{\circ} \mathrm{C}$. HeLa LC3 tandem were generated by stable transfection of Hela M cells with LC3 tandem vector mRFP-EGFP-LC3. HeLa TFEB/TFE3-KO was generated from Dr Youle R.J. from National Institutes of Health, Bethesda. U2OS LC3-GFP was provided by Dr Paolo Grumati (Tigem, Naples) and cultured in DMEM supplemented with $10 \%$ fetal bovine serum, $200 \mu \mathrm{M} \mathrm{L}$-glutamine, $5 \% \mathrm{CO}_{2}$ at $37^{\circ} \mathrm{C}$.

Cells were transfected for $24 \mathrm{~h}$ with the sequent plasmids: pDS-RED-C1-Beclin1 plasmid purchased at Addgene (Plasmid \#24405); TRPML1, TRPML2, and TRPML3 purchased at Origene (respectively plasmid number RC201010, RC211195, and RC224697); TRPML1-DDKK non-conducting pore mutant was a kind gift of professor Haoxing Xu from University of Michigan, TRPML1 GFP plasmid was generated by subcloning the TRPML1 coding sequence from RC201010 in a pEGFP-C3 plasmid. HeLa cells were transfected using TransIT ${ }^{\oplus}$ LT1 transfection reagent (Mirus) in accordance to the manufacturer's protocol; ARPE-19 cells were transfected using Lipofectamine LTX and Plus reagent (Thermo Fisher) in accordance to the manufacturer's protocol.

Cells were silenced with $40 \mathrm{nM}$ of siRNA against TRPML1 (sequence: $5^{\prime}$-CCUU CGCCGUCGUCUCAAA-3'); TRPML1 oligo \#2 (sequence: $5^{\prime}$-AUCCGAUGGUG GUUACUGA-3'); TRPML1 oligo \#3 (sequence: $5^{\prime}$-GAUCACGUUUGACAACAA A-3'); CaMKK $\beta$ (Sequence: 5'-GGCACAUCAAGAUCGCUGA-3'-oligo\#1 in Supplementary Fig. 6a, 5'-UGACAAUACCUACUAUGCA-3'-oligo\#2 in Supplementary Fig. 6a, 5'-CAAAGGCAUCGAGUACUUA-3'), CaMKI (5'-AUAC AGCUCUAGAUAAGAA-3' - oligo\#1 in Supplementary Fig. 6a, 5'-AGAUUUUG AAGGCCGAGUA- $3^{\prime}$-oligo\#2 in Supplementary Fig. 6a, $5^{\prime}$-CCAUAGGUGUCA UCGCCUA-3'), VPS34 (5'-GAGAUGUACUUGAACGUAA-3', 5'-GCAUGGAG AUGAUUUACGU-3', $5^{\prime}$-GCUUAGACCUGUCGGAUGA-3'), ULK1 (sequence: 5'-GCAUCGGCACCAUCGUCUA-3' -oligo\#1 in Supplementary Fig. 6a, 5'-GCA UGGACUUCGAUGAGUU-3'-oligo\#2 in Supplementary Fig. 6a, 5'-CGCCUGU UCUACGAGAAGA), ULK2 (5'-GCAGAUUAUUUGCAAGCGA-3' -oligo\#1 in Supplementary Fig. 6a, 5'-GCUCGUUACCUACAUAGUA-3', 5'-GAAUCUGA ACGAACGAUAU-3' - oligo\#2 in Supplementary Fig. 6a, ATG13 (L-020765-01 from Dharmacon), Beclin1 (siGENOME BECN1 siRNA - Pool Catalog Number M010552-01, from Dharmacon), TRPML2 (sequence: 5'-GGUUAUUUCCAGGCA UAUA-3', 5'-GCUCUAAGGUUACGGAAGA-3', 5'-GGAAUGCAGUAGCAAA GAA-3'), TRPML3 (sequence: 5'-CAUUAGAUCUGUGAUUAGA-3', 5'-GACU UUACUCUGACUAUAA-3', 5'-GAGUCUAACUAGUUAUGAU-3');

AMPK (sequence: 5'-GGAUCCAUCAUAUAGUUCA-3'-oligo\#1 in Supplementary Fig. 6a, 5'-GAGUCUACAGUUAUACCAA-3', 5'-CGGGAUCAG UUAGCAACUA- $3^{\prime}$-oligo\#2 in in Supplementary Fig. 6a), Beclin1 (Santa Cruz Biotechnology, Inc., BECN1 siRNA (h):sc-29797) - pool2 in Supplementary Fig. 6m), Syntaxin-17 (sequence: 5'-GGAGAAGAUUGACAGCAUU-3', 5'-CCUUUG ACCAGAUCCAUGA-3', 5'-GAGUUUGACUCAGAUAUAU-3') for $72 \mathrm{~h}$ using Lipofectamine RNAimax (Thermo Fisher) according to the manufacturer's protocol. In Supplementary Fig. 4 are reported all control experiment (qPCR or western blottings) performed for checking the reduction of gene expression in $\mathrm{KO}$ and silenced cells.

Generation of ARPE-19 CrispCa9. Generation of ARPE-19 CrispCa9 MCOLN1 KO cell lines. ARPE-19 (ATCC CCRL-2320) cells carrying a homozygous deletion of a C were generated by using the CRISPr/Cas9 system. The gRNA sequence (5'CCCTGCGACAAGTTTCGAGCCA-3') with low off-target score have been selected using the http://crispor.tefor.net/crispor.py tool. An "ALL in One" vector expressing Cas9, the specific gRNA, and GFP was obtained from SIGMA

(CAS9GFPP). The CAS9GFPP was nucleofected in ARPE-19 cells using the Amaxa Cat No VCA-1003 and transfected GFP-positive cells were fluorescence-activated cell sorted (FACS) into 96-well plates to obtain single-cell-derived colonies carrying the INDEL mutations. Upon genomic DNA extraction and DNA Sanger sequencing, a cell clone carrying the c.159delC was selected and expanded (Supplementary Fig. 9d). Generation of ARPE-19 CrispCa9 MCOLN2 KO cell line. ARPE-19 cell line was electroporated with plasmid encoding spCas 9 and sgRNA targeting exon 1 of the TRPML2 gene (sgRNA: 5'-TGGCTCGGTATTTTTCACAA GGG-3', in bold PAM sequence). Seven days post-electroporation, cells were analized by Surveyor Nuclease assay (Surveyor Mutation Detection kit, IDT Technology, catalog number 706020) to measure levels of NHEJ at TRPML2 locus. 2 weeks after electroporation single-cell clones were derived from bulk population through FACS sorting. Genomic DNA of individual clones were extracted and amplified with the following primers for the TRPML2 locus (F: 5'-CTTGTGGTAAGGGAAAAACCAA3'; R: 5'-GGAGAGGCTTTCCTGGATATTT-3'). PCR products were cloned in

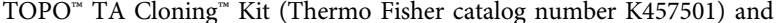
sequenced. Selected clone presented at sgRNA cutting site Indel/Mutationa at both alleles ( $\mathrm{T}$ insertion) that introduce a premature stop codon (Supplementary Fig. 9e). HAP-1 KO cells line: cell lines were purchased from Horizon. HAP-1 MCOLN1 KO: https://www.horizondiscovery.com/human-mcoln1-knockout-cellline-hzghc002537c011. Guide RNA sequence 5'-TGCGACAAGTTTCGAGCCAA3'. Targeted region: exon 2, mutation 5 bp deletion. HAP-1 MCOLN3 KO: https:// www.horizondiscovery.com/human-mcoln3-knockout-cell-line-122bp-deletion.
Guide RNA sequence: 5'-CAGCTATACAATGTCTCCGT-3'. Targeted region: exon 4, Mmutation 122 bp deletion.

Antibodies and western blotting. For western blottings, the following antibodies were used: B-Actin (Santa Cruz SC 47778, 1:4000), WIPI2 (Abcam, catalog number ab105459, 1:1000), LC3 (Novus NB100-2220, 1:1000), ULK1 (Cell Signalling, catalog number 8054 1:1000), Phospho-ULK1 (Ser757) (Cell Signalling, catalog number 6888 1:1000), Phospho-ULK1 (Ser555) (Cell Signalling, catalog number 5869 1:1000), p70 S6 Kinase (Cell Signalling, catalog number 2708 1:1000), Phospho-p70 S6 kinase (Thr389) (Cell Signalling, catalog number 9205 1:1000), Phospho-AMPKa (Thr172) (Cell Signalling, catalog number 2535 1:1000), AMPKa (Cell Signalling, catalog number 5831 1:1000), Phospho-ACC (Ser79) (Cell Signalling, catalog number 3661 1:1000), ACC (Cell Signalling, catalog number 3676 1:1000), CaMK1 (Abcam ab68234 1: 1000), CaMKK (Abcam Ab174289 1:1000), Phospho-BECLIN (Ser15) (Cell Signalling, catalog number 13825 1:1000), VINCULIN (Sigma, catalog number 9264), VPS34 (Z-R015, Echelon), VPS34 (Santa Cruz Biotechnology, Inc., Ab365404, 1:1000), ATG14 (Cell Signalling, catalog number 5504,1:1000), BECLIN1 (Cell Signalling, catalog number 3495, 1:1000), and Monoclonal ANTI-FLAG ${ }^{\oplus}$ M2 antibody (Sigma F3165 1:4000). Total cell lysate was prepared by solubilization in TRIS $\mathrm{HCl} 10 \mathrm{mM} \mathrm{pH} 8.0$ and $0.2 \%$ SDS supplemented with protein and phosphatases inhibitor (Sigma). Protein concentration was determined by the Bradford method. After SDS-polyacrylamide gel electrophoresis (PAGE) and immunoblotting, the protein recognized by the specific antibody were visualized by chemiluminescence methods (Luminata Crescendo Western HRP substrate, Millipore) using peroxidase-conjugated anti-rabbit or anti-mouse secondary antibodies (Millipore). Membranes were developed using a Chemidoc UVP imaging system (Ultra-Violet Products, Ltd) and densitometric quantification was performed in unsaturated images using ImageJ (NIH). All uncropped and unprocessed scans of western blottings are provided as Supplementary Fig. 11.

VPS34 immunoprecipitation and VPS34 kinase assay. HeLa cells were treated with DMSO (dimethylsulfoxide), MK6-83, or ML-SA1 for $30 \mathrm{~min}$. VPS34 immunoprecipitation was performed in accordance with the Echelon protocol (ZR015 from Echelon). Two milligrams of total protein lysate was incubated overnight with $2 \mu \mathrm{g} \mathrm{mg}^{-1}$ of protein of VPS34 antibody Z-R015. Day after immunoprecipitated complexes were incubated $2 \mathrm{~h}$ with protein A/G PLUS-Agarose (sc2003 Santa Cruz). The kinase assay was performed on VPS34 immunoprecipitated enzyme using the Class III PI3K Elisa Kit (K-3000 Echelon) following the instruction for beads conjugated enzyme. PI3P production was performed at $30^{\circ} \mathrm{C}$ for $40 \mathrm{~min}$ in constant shaking of 1100 r.p.m. Produced PI3P was collected by centrifugation from the beads and used for colorimetric detection on Elisa plate. Beads were resuspended in $35 \mu \mathrm{l}$ of $2 \times$ Laemmli Sample Buffer and boiled at $95^{\circ} \mathrm{C}$ for $5 \mathrm{~min}$, and run on SDS-PAGE to visualize the VPS34 complex by immunoblot.

Immunofluorescence. For immunofluorescence, the following antibodies were used: WIPI2 (Abcam, catalog number ab105459, 1:1000), Atg16L1 (D6D5, Cell Signaling, catalog number 8089, 1:200), LC3 (MBL PM036 1:400), LAMP1 (Santa Cruz, catalog number sc-20011, 1:400), TFEB (Cell Signalling, catalog number 4240 1:200), Phospho-S6 Ribosomal Protein (Ser235/236) (Cell Signalling, catalog number 9865 1:400), and FLAG M2 (Sigma, catalog number F3165). For WIPI2 and Atg16L1 staining, cells were fixed with ice-cold methanol for $5 \mathrm{~min}$ on ice and permeabilized in $0.1 \%(\mathrm{w} / \mathrm{v})$ saponin, $0.5 \%(\mathrm{w} / \mathrm{v})$ bovine serum albumin (BSA), and $50 \mathrm{mM} \mathrm{NH4Cl}$ in phosphate-buffered saline (PBS) (blocking buffer saponin). For LC3 and TFEB, cells were fixed in paraformaldehyde $4 \%$ for $20 \mathrm{~min}$. For LC3, cells were permeabilized with blocking buffer saponin, whereas for TFEB cells permeabilized in $0.1 \%(\mathrm{w} / \mathrm{v})$ Triton-X, $1 \%(\mathrm{w} / \mathrm{v})$ horse serum, and $1 \%(\mathrm{w} / \mathrm{v}) \mathrm{BSA}$ in PBS. Cells were incubated with the indicated primary antibodies for $1 \mathrm{~h}$ and subsequently incubated with secondary antibodies for $45 \mathrm{~min}$

The PX-594-conjugated antibody (1:200) was kindly provided by Dr. Ian Ganley from the University of Dundee. The staining was performed in accordance with the protocol described by Munson and Ganley ${ }^{44}$.

For confocal imaging, the samples were examined under a Zeiss LSM 800 confocal microscope. Optical sections were obtained under a $\times 63$ or $\times 40$ immersion objective at a definition of $1024 \times 1024$ pixels (average of 8 or 16 scans), adjusting the pinhole diameter to 1 Airy unit for each emission channel to have all the intensity values between 1 and 254 (linear range). For high-content images, we use OPERA system from PerkinElmer.

For image analysis, we used Columbus 2.6.0.127073 (built at 03:56 on 05/02/19) released by PerkinElmer. This online platform is based on Harmony High-Content Imaging and Analysis Software, which provides an easy quantification of complex cellular phenotypes. All the images were processed as follows: before starting the analysis sequence, the images were first segmented into the nuclei and cytoplasm using the Find Nuclei building block on the Hoechst channel and the Find Cytoplasm on the Cell Mask channel. To detect the spots (WIPI, LC3, and LAMP), the Find spots building block was applied to the spot channel. Then the Select population building block was used to select spots of certain intensity. The intensity 
and the morphology of each spot were calculated by adding the building blocks Calculate Intensity Properties and Calculate Morphology Properties. Finally, with define results building block we calculate the number of the spot for each cell.

Immuno-electron microscopy. Cells were fixed in a mixture of $4 \%$ paraformaldehyde and $0.05 \%$ glutaraldehyde prepared in $0.2 \mathrm{M}$ HEPES. Then, the cells were washed and incubated with the blocking/ permeabilizing mixture $(0.5 \% \mathrm{BSA}$, $0.1 \%$ saponin, $50 \mathrm{mM} \mathrm{NH}_{4} \mathrm{Cl}$ ) for $30 \mathrm{~min}$ and subsequently with the primary antibody against WIPI2, diluted 1:100 in blocking/permeabilizing solution, overnight. The following day the cells were washed and incubated with the secondary antibody, the anti-rabbit Fab' fragment coupled to $1.4 \mathrm{~nm}$ gold particles, diluted 1:50 in blocking/permeabilizing solution, for $2 \mathrm{~h}$. After this incubation with the antibodies, the gold-enhancement reaction has to be performed to increase the size of the $1.4 \mathrm{~nm}$ gold particles. Then samples were post-fixed in a mixture of osmium tetroxide and potassium ferrocyanide, dehydrated in ethanol and acetone, and embedded in epoxy resin as described previously (Polishchuk et al. ${ }^{61}$ ). Thin, $60 \mathrm{~nm}$ serial sections were cut using a Leica EM UC7 ultramicrotome. Electron microscopic images were acquired using a FEI Tecnai-12 electron microscope (FEI, Eindhoven, Netherlands) equipped with a VELETTA CCD digital camera (Soft Imaging Systems GmbH, Munster, Germany).

RNA extraction and qPCR. Total RNA was extracted from cells using RNeasy Plus Mini Kit (Qiagen). Reverse transcription was performed using QuantiTect Rev Transcription Kit (Qiagen). Real-time quantitative reverse-transcription PCR (qRT-PCR) was performed using the LightCycler ${ }^{\circledast}$ System 2.0 (Roche Applied Science). HPRT was used for qRT-PCR as reference gene. The parameters of realtime qRT-PCR amplification were according to Roche recommendations. The following primers were used in this study: HPRT fw: $5^{\prime}$-TGGCGTCGTGATTAG TGATG-3', rev: 5'-AACACCCTTTCCAAATCCTCA-3'; TRPML1 fw: 5'-GAGTG GGTGCGACAAGTTTC-3', rev: 5'-TGTTCTCTTCCCGGAATGTC-3'; WIPI2 fw: $5^{\prime}$-TACCTGCCTTCCCAAGTGAC-3', rev: 5'-AGCGAGCAGATGTTTTTGT G-3', ATP6VOE1; fw: 5' -cattgtgatgagcgtgttctgg-3', rev: 5'-AACTCCCCGGTTAGG ACCCTTA-3', ATP6V1H; fw: 5'-GGAAGTGTCAGATGATCCCCA-3', rev: 5'-CC GTTTGCCTCGTGGATAAT-3', TFEB; fw: 5'-CAAGGCCAATGACCTGGAC-3', rev: 5'-AGCTCCCTGGACTTTTGCAG-3', DPP7; fw: 5'-GATTCGGAGGAACCT GAGTG-3', rev: 5'-CGGAAGCAGGATCTTCTGG-3', TPP1; fw: 5'-GATCCCAG CTCTCCTCAATAC-3', rev: 5'-GCCATTTTTGCACCGTGTG-3', NEU1; fw: $5^{\prime}$-T GAAGTGTTTGCCCCTGGAC-3', rev: 5'-AGGCACCATGATCATCGCTG-3'; ATG135'-GACCTTCTATCGGGAGTTTCAG-3', rev: 5'-GGGTTTCCACAAAGG CATCAAC-3'; ULK1 fw: 5'-AGGTGGCCGAGCTACTGTC-3', rev: 5'-GGCCTT GTACACCTCATTC-3'; ULK2 fw: 5'-GGTTGGTACCSTTCCTGAGC-3'; rev: 5'-AGAGACTGTGGGGACTGAG-3'; Beclin1 fw: 5'-AGCCCCTGAAACTGGAC AC-3', rev: 5'-TCTTCCTCCTGGGTCTCTCC-3'; CaMK1 fw: 5'-TGTCATCGCC TACATCTTGC-3', rev: 5'-CAAACTCGTACTCGGCCTTC-3'; CaMKK $\beta$ fw: 5'-T CAAACCTTCCAACCTCCTG, rev: 5'-TTGCTCACACCAAAGTCAGC-3'; TRPML3 fw: 5'-GAAGTTCTGGGCTCGAGGTAG-3', rev: 5'-GATTCCCAACG GAGACATTG-3'; TRPML2 fw: 5'-ATGGCACATCGTGATTCTGA-3', rev: 5'-C GTCTGGCTCGGTATTTTTC-3'; AMPK $\alpha$ fw: 5'-GTATGCAGGCCCAGAGGT AG-3', rev: 5'-GAGTTGGCACATGGTCATCA-3'; MAP1 fw: 5'-CGGAGAAGAC CTTCAAGCAC-3', rev: 5'-CTGCTTCTCACCCTTGTATCG-3'.

Statistics and reproducibility. Sample sizes and reproducibility for each figure are denoted in the figure legends. Immunofluorescence experiments were repeated independently 3 times and at least 50 cells were analyzed from a single experiment. Representative images are shown in Figs. 1a, b, d, e, 2a-e, 3a-e, 4a-d, f, g, 5a, and 6a-e, and in Supplementary Figs. 1, 2a, b, e, 3, 4b, c, e, f, h, 5a, c, d, 6a, m, 7a-e, $8 \mathrm{a}-\mathrm{c}, 9$, and $10 \mathrm{c}$. Western blotting and immunoprecipitation experiments were repeated at least three times and representative blots are shown in Figs. 1c, 4e, h, i, and 5b, c, and in Supplementary Figs. 2c, d, 3a, 4e-g, 5b, e, 6l, 7f, g, and 10a. Most data are presented as the means \pm SD unless otherwise specified. Statistical comparisons were made using Student's $t$-test when comparing two groups.

\section{Data availability}

The datasets generated and/or analyzed during the current study are available from the corresponding author on reasonable request. The source data underlying Figs. 1a, b, d, e, 2, 3, 4a-d, f, g, 5a, and 6a-f are provided as a Source Data file.

Received: 21 November 2018; Accepted: 14 November 2019; Published online: 10 December 2019

\section{References}

1. Berridge, M. J., Lipp, P. \& Bootman, M. D. The versatility and universality of calcium signalling. Nat. Rev. Mol. Cell Biol. 1, 11-21 (2000).
2. La Rovere, R. M. et al. Intracellular $\mathrm{Ca}(2+)$ signaling and $\mathrm{Ca}(2+)$ microdomains in the control of cell survival, apoptosis and autophagy. Cell Calcium 60, 74-87 (2016).

3. Xu, H. \& Ren, D. Lysosomal physiology. Annu. Rev. Physiol. 77, 57-80 (2015).

4. Morgan, A. J. et al. Molecular mechanisms of endolysosomal Ca2+ signalling in health and disease. Biochem. J. 439, 349-374 (2011).

5. Shen, D. et al. Lipid storage disorders block lysosomal trafficking by inhibiting a TRP channel and lysosomal calcium release. Nat. Commun. 3, 731 (2012).

6. Lloyd-Evans, E. et al. Niemann-Pick disease type $\mathrm{C} 1$ is a sphingosine storage disease that causes deregulation of lysosomal calcium. Nat. Med. 14, 1247-1255 (2008).

7. Coen, K. et al. Lysosomal calcium homeostasis defects, not proton pump defects, cause endo-lysosomal dysfunction in PSEN-deficient cells. J. Cell Biol. 198, 23-35 (2012).

8. Funk, K. E. \& Kuret, J. Lysosomal fusion dysfunction as a unifying hypothesis for Alzheimer's disease pathology. Int. J. Alzheimers Dis. 2012, 752894 (2012).

9. Bae, E. J. et al. Phospholipase D1 regulates autophagic flux and clearance of alpha-synuclein aggregates. Cell Death Differ. 21, 1132-1141 (2014).

10. Ezeani, M. \& Omabe, M. A new perspective of lysosomal cation channeldependent homeostasis in Alzheimer's disease. Mol. Neurobiol. 53, 1672-1678 (2016).

11. Feng, X. \& Yang, J. Lysosomal calcium in neurodegeneration. Messenger (Los Angel) 5, 56-66 (2016).

12. Chen, M. et al. Association between soy isoflavone intake and breast cancer risk for pre- and post-menopausal women: a meta-analysis of epidemiological studies. PLoS ONE 9, e89288 (2014).

13. Kondratskyi, A. et al. Calcium-permeable ion channels in control of autophagy and cancer. Front. Physiol. 4, 272 (2013).

14. Galluzzi, L. et al. Autophagy in malignant transformation and cancer progression. EMBO J. 34, 856-880 (2015).

15. Bassi, M. T. et al. Cloning of the gene encoding a novel integral membrane protein, mucolipidin-and identification of the two major founder mutations causing mucolipidosis type IV. Am. J. Hum. Genet. 67, 1110-1120 (2000).

16. Frei, K. P. et al. Mucolipidosis type IV: characteristic MRI findings. Neurology 51, 565-569 (1998)

17. Berman, E. R. et al. Congenital corneal clouding with abnormal systemic storage bodies: a new variant of mucolipidosis. J. Pediatr. 84, 519-526 (1974).

18. Curcio-Morelli, C. et al. Macroautophagy is defective in mucolipin-1-deficient mouse neurons. Neurobiol. Dis. 40, 370-377 (2010).

19. Vergarajauregui, S. et al. Autophagic dysfunction in mucolipidosis type IV patients. Hum. Mol. Genet. 17, 2723-2737 (2008).

20. Di Paola, S., Scotto-Rosato, A. \& Medina, D. L. TRPML1: The Ca((2+))retaker of the lysosome. Cell Calcium 69, 112-121 (2018).

21. Lieberman, A. P. et al. Autophagy in lysosomal storage disorders. Autophagy 8, 719-730 (2012)

22. Medina, D. L. \& Ballabio, A. Lysosomal calcium regulates autophagy. Autophagy 11, 970-971 (2015).

23. Medina, D. L. et al. Lysosomal calcium signalling regulates autophagy through calcineurin and TFEB. Nat. Cell Biol. 17, 288-99. (2015).

24. Venkatachalam, K., Wong, C. O. \& Zhu, M. X. The role of TRPMLs in endolysosomal trafficking and function. Cell Calcium 58, 48-56 (2015).

25. Ahuja, M. et al. TRPML1 as lysosomal fusion guard. Channels (Austin) 10, 261-263 (2016).

26. Settembre, C. et al. TFEB links autophagy to lysosomal biogenesis. Science 332, 1429-1433 (2011)

27. Ben-Ari, Y. et al. The life of an mRNA in space and time. J. Cell Sci. 123, 1761-1774 (2010)

28. Wang, W. et al. Up-regulation of lysosomal TRPML1 channels is essential for lysosomal adaptation to nutrient starvation. Proc. Natl Acad. Sci. USA 112, E1373-E1381 (2015).

29. Chen, C. C. et al. A small molecule restores function to TRPML1 mutant isoforms responsible for mucolipidosis type IV. Nat. Commun. 5, 4681 (2014).

30. Plesch, E. et al. Selective agonist of TRPML2 reveals direct role in chemokine release from innate immune cells. Elife 7, e39720 (2018).

31. Grimm, C. et al. Small molecule activators of TRPML3. Chem. Biol. 17, 135-148 (2010).

32. Li, X. et al. A molecular mechanism to regulate lysosome motility for lysosome positioning and tubulation. Nat. Cell Biol. 18, 404-417 (2016).

33. Klionsky, D. J. et al. Guidelines for the use and interpretation of assays for monitoring autophagy in higher eukaryotes. Autophagy 4, 151-175 (2008).

34. De Leo, M. G. et al. Autophagosome-lysosome fusion triggers a lysosomal response mediated by TLR9 and controlled by OCRL. Nat. Cell Biol. 18, 839-850 (2016).

35. Jacquin, E. et al. Pharmacological modulators of autophagy activate a parallel noncanonical pathway driving unconventional LC3 lipidation. Autophagy 13 854-867 (2017).

36. Nezich, C. L. et al. MiT/TFE transcription factors are activated during mitophagy downstream of Parkin and Atg5. J. Cell Biol. 210, 435-50. (2015). 
37. Sun, I. H. et al. mTOR complex 1 signaling regulates the generation and function of central and effector Foxp3(+) regulatory T cells. J. Immunol. 201, 481-492 (2018).

38. Axe, E. L. et al. Autophagosome formation from membrane compartments enriched in phosphatidylinositol 3-phosphate and dynamically connected to the endoplasmic reticulum. J. Cell Biol. 182, 685-701 (2008).

39. Proikas-Cezanne, T. et al. WIPI proteins: essential PtdIns3P effectors at the nascent autophagosome. J. Cell Sci. 128, 207-17. (2015).

40. Polson, H. E. et al. Mammalian Atg18 (WIPI2) localizes to omegasomeanchored phagophores and positively regulates LC3 lipidation. Autophagy 6, 506-22. (2010).

41. Marat, A. L. \& Haucke, V. Phosphatidylinositol 3-phosphates-at the interface between cell signalling and membrane traffic. EMBO J. 35, 561-79. (2016).

42. Ronan, B. et al. A highly potent and selective Vps34 inhibitor alters vesicle trafficking and autophagy. Nat. Chem. Biol. 10, 1013-1019 (2014).

43. Kanai, F. et al. The PX domains of p47phox and p40phox bind to lipid products of PI(3)K. Nat. Cell Biol. 3, 675-678 (2001)

44. Munson, M. J. \& Ganley, I. G. Determination of cellular phosphatidylinositol3-phosphate (PI3P) levels using a fluorescently labelled selective PI3P binding domain (PX). Bio Protoc. 6, e1903 (2016).

45. Mizushima, N., Yoshimori, T. \& Levine, B. Methods in mammalian autophagy research. Cell 140, 313-326 (2010).

46. Stephan, J. S. et al. The Tor and PKA signaling pathways independently target the Atg1/Atg13 protein kinase complex to control autophagy. Proc. Natl Acad. Sci. USA 106, 17049-17054 (2009).

47. Alers, S., Wesselborg, S. \& Stork, B. ATG13: just a companion, or an executor of the autophagic program?. Autophagy 10, 944-956 (2014).

48. Kamada, Y. et al. Tor directly controls the Atg1 kinase complex to regulate autophagy. Mol. Cell. Biol. 30, 1049-58. (2010)

49. Kamada, Y. et al. Tor-mediated induction of autophagy via an Apg1 protein kinase complex. J. Cell Biol. 150, 1507-1513 (2000).

50. Cao, Q. et al. The lysosomal $\mathrm{Ca}(2+)$ release channel TRPML1 regulates lysosome size by activating calmodulin. J. Biol. Chem. 292, 8424-8435 (2017).

51. Hoyer-Hansen, M. et al. Control of macroautophagy by calcium, calmodulin-dependent kinase kinase-beta, and Bcl-2. Mol. Cell 25, 193-205 (2007).

52. Woods, A. et al. Ca2+/calmodulin-dependent protein kinase kinase-beta acts upstream of AMP-activated protein kinase in mammalian cells. Cell. Metab. 2, 21-33 (2005)

53. Hawley, S. A. et al. Calmodulin-dependent protein kinase kinase-beta is an alternative upstream kinase for AMP-activated protein kinase. Cell. Metab. 2, 9-19 (2005).

54. Egan, D. F. et al. Phosphorylation of ULK1 (hATG1) by AMP-activated protein kinase connects energy sensing to mitophagy. Science 331, 456-461 (2011).

55. Kim, J. et al. AMPK and mTOR regulate autophagy through direct phosphorylation of Ulk1. Nat. Cell Biol. 13, 132-141 (2011).

56. Mao, K. \& Klionsky, D. J. AMPK activates autophagy by phosphorylating ULK1. Circ. Res. 108, 787-788 (2011).

57. Ha, J. et al. Critical phosphorylation sites for acetyl-CoA carboxylase activity. J. Biol. Chem. 269, 22162-22168 (1994).

58. Funderburk, S. F., Wang, Q. J. \& Yue, Z. The Beclin 1-VPS34 complex-at the crossroads of autophagy and beyond. Trends Cell Biol. 20, 355-62. (2010).

59. Nazarko, V. Y. \& Zhong, Q. ULK1 targets Beclin-1 in autophagy. Nat. Cell Biol. 15, 727-728 (2013).

60. Bargal, R. et al. Identification of the gene causing mucolipidosis type IV. Nat. Genet. 26, 118-23. (2000).

61. Polishchuk, E. V. et al. Wilson disease protein ATP7B utilizes lysosomal exocytosis to maintain copper homeostasis. Dev. Cell. 29, 686-700 (2014).

62. Cheng, X. et al. Mucolipins: Intracellular TRPML1-3 channels. FEBS Lett. 584, 2013-2021 (2010).

63. Samie, M. et al. A TRP channel in the lysosome regulates large particle phagocytosis via focal exocytosis. Dev. Cell. 26, 511-524 (2013).

64. Kiselyov, K. et al. TRPML: transporters of metals in lysosomes essential for cell survival? Cell Calcium 50, 288-294 (2011).

65. Medina, D. L. et al. Transcriptional activation of lysosomal exocytosis promotes cellular clearance. Dev. Cell. 21, 421-430 (2011).

66. $\mathrm{Xu}, \mathrm{H}$. et al. Activating mutation in a mucolipin transient receptor potential channel leads to melanocyte loss in varitint-waddler mice. Proc. Natl Acad. Sci. USA 104, 18321-18326 (2007).

67. Waller-Evans, H. \& Lloyd-Evans, E. Regulation of TRPML1 function. Biochem. Soc. Trans. 43, 442-446 (2015).

68. Dong, X. P. et al. PI $(3,5) \mathrm{P}(2)$ controls membrane trafficking by direct activation of mucolipin $\mathrm{Ca}(2+)$ release channels in the endolysosome. Nat. Commun. 1, 38 (2010).
69. Zhang, S. et al. Cryo-EM structures of the mammalian endo-lysosomal TRPML1 channel elucidate the combined regulation mechanism. Protein Cell 8, 834-847 (2017)

70. Wang, W. et al. TRPML1: an ion channel in the lysosome. Handb. Exp. Pharmacol. 222, 631-45. (2014).

71. Kim, M. S. et al. Exosomal release through TRPML1-mediated lysosomal exocytosis is required for adipogenesis. Biochem. Biophys. Res. Commun. 510 409-415 (2019).

72. Cheng, $\mathrm{X}$. et al. The intracellular $\mathrm{Ca}(2)(+)$ channel MCOLN1 is required for sarcolemma repair to prevent muscular dystrophy. Nat. Med. 20, 1187-1192 (2014).

73. Zhang, X. et al. Rapamycin directly activates lysosomal mucolipin TRP channels independent of mTOR. PLoS Biol. 17, e3000252 (2019).

74. Sun, X. et al. A negative feedback regulation of MTORC1 activity by the lysosomal $\mathrm{Ca}(2+)$ channel MCOLN1 (mucolipin 1) using a CALM (calmodulin)-dependent mechanism. Autophagy 14, 38-52 (2018).

75. Zhang, X. et al. MCOLN1 is a ROS sensor in lysosomes that regulates autophagy. Nat. Commun. 7, 12109 (2016).

76. Palmieri, M. et al. Characterization of the CLEAR network reveals an integrated control of cellular clearance pathways. Hum. Mol. Genet. 20, 3852-66. (2011)

77. Chen, C. C. et al. Small molecules for early endosome-specific patch clamping. Chem. Biol. 24, 907-916 e4 (2017).

78. Zeevi, D. A. et al. Heteromultimeric TRPML channel assemblies play a crucial role in the regulation of cell viability models and starvation-induced autophagy. J. Cell Sci. 123, 3112-3124 (2010).

79. Choi, S. H. et al. A three-dimensional human neural cell culture model of Alzheimer's disease. Nature 515, 274-278 (2014).

80. Kim, $\mathrm{H}$. J. et al. The $\mathrm{Ca}(2+)$ channel TRPML3 regulates membrane trafficking and autophagy. Traffic 10, 1157-67. (2009).

81. Martina, J. A., Lelouvier, B. \& Puertollano, R. The calcium channel mucolipin3 is a novel regulator of trafficking along the endosomal pathway. Traffic 10, 1143-56. (2009)

82. Zeevi, D. A. et al. A potentially dynamic lysosomal role for the endogenous TRPML proteins. J. Pathol. 219, 153-162 (2009).

83. Lamb, C. A., Yoshimori, T. \& Tooze, S. A. The autophagosome: origins unknown, biogenesis complex. Nat. Rev. Mol. Cell Biol. 14, 759-774 (2013).

84. Gulati, P. et al. Amino acids activate mTOR complex 1 via $\mathrm{Ca} 2+/ \mathrm{CaM}$ signaling to hVps34. Cell. Metab. 7, 456-465 (2008).

85. Joyal, J. L. et al. Calmodulin activates phosphatidylinositol 3-kinase. J. Biol. Chem. 272, 28183-28186 (1997)

86. Pfisterer, U. et al. Direct conversion of human fibroblasts to dopaminergic neurons. Proc. Natl Acad. Sci. USA 108, 10343-10348 (2011).

87. Shang, L. et al. Nutrient starvation elicits an acute autophagic response mediated by Ulk1 dephosphorylation and its subsequent dissociation from AMPK. Proc. Natl Acad. Sci. USA 108, 4788-4793 (2011).

88. Russell, R. C. et al. ULK1 induces autophagy by phosphorylating Beclin-1 and activating VPS34 lipid kinase. Nat. Cell Biol. 15, 741-50. (2013).

89. Alers, S. et al. Role of AMPK-mTOR-Ulk1/2 in the regulation of autophagy: cross talk, shortcuts, and feedbacks. Mol. Cell. Biol. 32, 2-11 (2012).

90. Di Malta, C. et al. Transcriptional activation of RagD GTPase controls mTORC1 and promotes cancer growth. Science 356, 1188-1192 (2017).

\section{Acknowledgements}

We acknowledge financial support from Italian Telethon Foundation (TMDMHCSIITT) (D.L.M.), Mucolipidosis Type IV Foundation (D.L.M. and A.S.R.), Horizon 2020 grant BATCure (666918) (D.L.M. and C.S.). A.B. was supported by grants from the Italian Telethon Foundation (TGM16CB6), MIUR FIRB RBAP11Z3YA, European Research Council Advanced Investigator number 694282 (LYSOSOMICS), and U.S. National Institutes of Health (R01-NS078072). C.G. was supported by funding from the German Research Foundation and DFG (project number 239283807, SFB/TRR152 project P04) We thank Graciana Diez-Roux, Carmine Settembre, and Roman Polishchuk for the helpful discussion and comments during the preparation of the manuscript. We thank Nicholas Ktistakis for the HEK293-GFP-DFCP1. We thank Dr Paolo Grumati for U2OS LC3-GFP cells. We thank Dr Ian Ganley for the PX recombinant probe.

\section{Author contributions}

D.L.M. conceived the project. A.S.R., S.M., C.S., S.D.P., A.C., J.M., E.P., M.A.D., A.B., and D.L.M. designed experiments. A.S.R., S.M., C.S., S.D.P., A.C., J.M., and E.P. performed experiments. C.G. provided selective agonist for various TRPMLs. A.A. and A.L. generate TRPML2 KO cells. S.D.P. generated Fig. 7. D.L.M. wrote the manuscript.

\section{Competing interests}

A.B. is a founder of Casma Therapeutics, Inc. The remaining authors declare no competing interests. 


\section{Additional information}

Supplementary information is available for this paper at https://doi.org/10.1038/s41467019-13572-w.

Correspondence and requests for materials should be addressed to D.L.M.

Peer review information Nature Communication thanks Jan Parys and other, anonymous, reviewers for their contributions to the peer review of this work.

Reprints and permission information is available at http://www.nature.com/reprints

Publisher's note Springer Nature remains neutral with regard to jurisdictional claims in published maps and institutional affiliations. (c) Open Access This article is licensed under a Creative Commons Attribution 4.0 International License, which permits use, sharing, adaptation, distribution and reproduction in any medium or format, as long as you give appropriate credit to the original author(s) and the source, provide a link to the Creative Commons license, and indicate if changes were made. The images or other third party material in this article are included in the article's Creative Commons license, unless indicated otherwise in a credit line to the material. If material is not included in the article's Creative Commons license and your intended use is not permitted by statutory regulation or exceeds the permitted use, you will need to obtain permission directly from the copyright holder. To view a copy of this license, visit http://creativecommons.org/ licenses/by/4.0/.

(C) The Author(s) 2019 